\title{
Minimal Unitary Realizations of Exceptional U-duality Groups and Their Subgroups as Quasiconformal Groups ${ }^{1}$
}

\author{
M. Günaydin and O. Pavlyk \\ Penn State University, \\ Physics Department, \\ University Park, PA 16802 \\ E-mails: murat@phys.psu.edu; pavlyk@phys.psu.edu
}

\begin{abstract}
We study the minimal unitary representations of noncompact exceptional groups that arise as U-duality groups in extended supergravity theories. First we give the unitary realizations of the exceptional group $\mathrm{E}_{8(-24)}$ in $\mathrm{SU}^{*}(8)$ as well as $\mathrm{SU}(6,2)$ covariant bases. $\mathrm{E}_{8(-24)}$ has $\mathrm{E}_{7} \times \mathrm{SU}(2)$ as its maximal compact subgroup and is the U-duality group of the exceptional supergravity theory in $d=3$. For the corresponding $\mathrm{U}$-duality group $\mathrm{E}_{8(8)}$ of the maximal supergravity theory the minimal realization was given in hep-th/0109005 The minimal unitary realizations of all the lower rank noncompact exceptional groups can be obtained by truncation of those of $\mathrm{E}_{8(-24)}$ and $\mathrm{E}_{8(8)}$. By further truncation one can obtain the minimal unitary realizations of all the groups of the "Magic Triangle". We give explicitly the minimal unitary realizations of the exceptional subgroups of $\mathrm{E}_{8(-24)}$ as well as other physically interesting subgroups. These minimal unitary realizations correspond, in general, to the quantization of their geometric actions as quasi-conformal groups as defined in hep-th/0008063.
\end{abstract}

\section{Introduction}

The concept of a minimal unitary representation of a non-compact group $G$ was first introduced by A. Joseph [1]. It is defined as a unitary representation on a Hilbert space of functions depending on the minimal number of coordinates for a given non-compact group. By introducing position operators corresponding to these coordinates and the momenta conjugate to them, one obtains the minimal realization by expressing the generators of the Lie algebra $\mathfrak{g}$ of $G$ in terms of these canonical operators. Joseph's main motivation was to give a general mathematical framework for spectrum generating symmetry algebras that were studied by physicists earlier [2].

The minimal realization of complex forms of classical Lie algebras and of $\mathfrak{g}_{2(2)}$ were given by Joseph [1, 3] in a Cartan-Weyl basis. Over the last two decades there has been an ever increasing interest by the mathematicians on the minimal unitary representations of noncompact groups. For a review and

\footnotetext{
${ }^{1}$ Work supported in part by the National Science Foundation under grant number PHY0245337.
} 
the references on earlier work on the subject we refer the reader to the review lectures of Jian-Shu Li 4 .

More recently, minimal unitary representations were studied by Pioline, Kazhdan and Waldron [5] and by Günaydin, Koepsell and Nicolai [6] with the goal of applying them to M-theory. The work of KPW was motivated the idea that the theta series of $\mathrm{E}_{8(8)}$ and its subgroups may describe the quantum supermembrane in various dimensions [7]. On the other hand the work of GKN was motivated by the idea that the spectra of M-theory in various dimensions must fall into unitary representations of its U-duality group in the respective dimensions. Realization of the minimal unitary representation of $\mathrm{E}_{8(8)}$ and its subalgebras given in [6] is based on an earlier work [8] of the authors on geometric realization of $\mathrm{E}_{8(8)}$ as a quasi-conformal group acting on a 57-dimensional space with a quartic norm form.

The groups $\mathrm{E}_{6(6)}, \mathrm{E}_{7(7)}$ and $\mathrm{E}_{8(8)}$ arise as U-duality groups of maximal supergravity theories obtained from the 11 dimensional supergravity [9] by toroidal compactification to $d=5,4$ and $d=3$ dimensions, respectively. For M-theory that has the 11 dimensional supergravity as its low energy effective theory in a strongly coupled phase one expects only the discrete subgroups $\mathrm{E}_{6(6)}(\mathbb{Z})$, $\mathrm{E}_{7(7)}(\mathbb{Z})$ and $\mathrm{E}_{8(8)}(\mathbb{Z})$ to be symmetries of the full nonperturbative theory. Hence we expect the spectra of M-theory to fall into unitary representations of these discrete subgroups of U-duality groups in the respective compactifications.

As was shown in 8$] \mathrm{E}_{8(8)}$ has a natural action as a quasi-conformal group in the charge-entropy space of BPS black hole solutions in $N=8$ supergravity in $d=4$ and hence can be interpreted as its spectrum generating symmetry group. The formula relating the entropy of a four dimensional BPS black hole solution to its charges defines a generalized light-cone in the charge-entropy space which is left invariant by the quasiconformal group action of $\mathrm{E}_{8(8)}$.

In addition to $\mathrm{E}_{8(8)}$ the only other non-compact real form of $\mathrm{E}_{8}$ is $\mathrm{E}_{8(-24)}$ whose maximal compact subgroup is $\mathrm{E}_{7} \otimes \mathrm{SU}(2)$. It is the U-duality group of the exceptional $\mathcal{N}=4$ supergravity in $d=3$, which can be obtained, by dimensional reduction, from the exceptional $\mathcal{N}=2$ Maxwell-Einstein supergravity theory (MESGT) in $d=5$ describing the coupling of 26 vector multiplets to pure $\mathcal{N}=2$ supergravity [10]. In five dimensions U-duality group of the exceptional MESGT is $\mathrm{E}_{6(-26)}$ with maximal compact subgroup $\mathrm{F}_{4}$ and in $d=4$ it is $\mathrm{E}_{7(-25)}$ with maximal compact subgroup $\mathrm{E}_{6} \otimes \mathrm{U}(1)$.

In this paper we extend the results of [ $[6]$ on the minimal unitary representations of $\mathrm{E}_{8(8)}$ to the other noncompact real form $\mathrm{E}_{8(-24)}$ and study their truncations to other exceptional quasi-conformal subalgebras as well as other classical subalgebras. We should note that, just like $\mathrm{E}_{8(8)}$, the group $\mathrm{E}_{8(-24)}$ can be realized as a quasi-conformal group acting on a 57-dimensional space. For the exceptional $\mathcal{N}=2 d=4$ supergravity theory this 57 -dimensional space is the charge-entropy space and $\mathrm{E}_{8(-24)}$ acts as a spectrum generating quasiconformal symmetry that leaves the generalized light-cones invariant.

The plan of the paper is as follows. In section 2 we write down the Lie algebra of the exceptional group $\mathrm{E}_{8(-24)}$ and then give its minimal unitary real- 
ization in a $\mathrm{SU}^{*}(8)$ as well as $\mathrm{SU}(6,2)$ covariant bases. The $\mathrm{SU}^{*}(8)$ basis is the coordinate basis (Schrödinger picture) while the $\mathrm{SU}(6,2)$ basis is the oscillator realization. They correspond to the $\mathrm{SL}(8, \mathbb{R})$ and $\mathrm{SU}(8)$ bases of the maximally split exceptional group $\mathrm{E}_{8(8)}$ with maximal compact subgroup $S O(16)$ given in 6]. In section 3 we give the minimal unitary realizations of $E_{7(-5)}$ with maximal compact subgroup $\mathrm{SO}(12) \times \mathrm{SU}(2), \mathrm{E}_{6(2)}$ with maximal compact subgroup $\mathrm{SU}(6) \times \mathrm{SU}(2), \mathrm{E}_{6(-14)}$ with the maximal compact subgroup $\mathrm{SO}(10) \times \mathrm{U}(1)$, $\mathrm{F}_{4(4)}$ with maximal compact subgroup $\mathrm{USp}(6) \times \mathrm{USp}(2)$ and $\mathrm{SO}(4,4)$ by consistent truncation of the minimal unitary realization of $\mathrm{E}_{8(-24)}$. In section 4 we study a different chain of truncations and give the minimal unitary realization of $\mathrm{E}_{7(-25)}$ with maximal compact subgroup $E_{6} \times \mathrm{U}(1)$ and of $\mathrm{SO}(2 p, 2)$ for $p=2,3,4,5$. We conclude with a discussion of our results and future directions. In appendix A we give the explicit transformations for going from the $\mathrm{SU}^{*}(8)$ basis to the $\mathrm{SU}(6,2)$ of the minimal realization of $\mathrm{E}_{8(-24)}$ and in appendix $\mathrm{B}$ we give the minimal unitary realization of $\mathrm{E}_{8(8)}$ in a $\mathrm{SU}^{*}(8)$ covariant basis.

\section{Minimal Unitary Representation of $\mathrm{E}_{8(-24)}$}

The Lie algebra $\mathfrak{e}_{8(-24)}$ of $\mathrm{E}_{8(-24)}$ admits a 5-grading with respect its subalgebra $\mathfrak{e}_{7(-25)} \oplus \mathfrak{s o}(1,1)$ determined by the generator $\Delta$ of a dilatation subgroup $S O(1,1)$

$$
\mathfrak{e}_{8(-24)}=\begin{array}{ccccccccc}
\mathfrak{g}^{-2} & \oplus & \mathfrak{g}^{-1} & \oplus & \mathfrak{g}^{0} & \oplus & \mathfrak{g}^{+1} & \oplus & \mathfrak{g}^{+2} \\
\mathbf{1} & \oplus & \mathbf{5 6} & \oplus & (\mathbf{1 3 3} \oplus \mathbf{1}) & \oplus & \mathbf{5 6} & \oplus & \mathbf{1}
\end{array}
$$

such that $\mathfrak{g}^{ \pm 2}$ generators together with $\Delta$ form an $\mathfrak{s l}(2, \mathbb{R})$ subalgebra.

To construct the minimal unitary representation of $\mathfrak{e}_{8(-24)}$ we find it convenient to work in a basis covariant with respect to $\mathfrak{s u}^{*}(8)$ subalgebra of $\mathfrak{e}_{7(-25)}{ }^{2}$. In the $\mathfrak{s u}^{*}(8)$ basis the generators of $\mathfrak{e}_{7(-25)}$ can be labelled as follows

$$
\mathbf{1 3 3}=\mathbf{6 3} \oplus \mathbf{7 0}=J^{A}{ }_{B} \oplus J^{A B C D}
$$

where $J^{A}{ }_{B}$ denote the generators of $\mathfrak{s u}^{*}(8)$ and $J^{A B C D}$ is completely antisymmetric in its indices $A, B, \ldots=1,2, \ldots, 8$. They satisfy the commutation relations

$$
\begin{aligned}
{\left[J^{A}{ }_{B}, J^{C}{ }_{D}\right] } & =\delta^{C}{ }_{B} J^{A}{ }_{D}-\delta^{A}{ }_{D} J^{C}{ }_{B} \\
{\left[J^{A}{ }_{B}, J^{C D E F}\right] } & =-4 \delta^{[C}{ }_{B} J^{D E F] A}-\frac{1}{2} \delta^{A}{ }_{B} J^{C D E F} \\
{\left[J^{A B C D}, J^{E F G H}\right] } & =-\frac{1}{36} \epsilon^{A B C D K[E F G} J^{H]}{ }_{K}
\end{aligned}
$$

and the following reality conditions

$$
\begin{aligned}
\left(J_{B}^{A}\right)^{\dagger} & =J_{A}{ }^{B}=\Omega_{A C} \Omega^{B D} J^{C}{ }_{D} \\
\left(J^{A B C D}\right)^{\dagger} & =-J_{A B C D}=-\Omega_{A E} \Omega_{B F} \Omega_{C G} \Omega_{D H} J^{E F G H}
\end{aligned}
$$

\footnotetext{
${ }^{2}$ The $\mathfrak{s u}^{*}(8)$-covariant basis of $\mathfrak{e}_{7(-25)}$ is the analog of $\mathfrak{s l}(8, \mathbb{R})$ basis of $\mathfrak{e}_{7(7)} 8$
} 
where $\Omega$ is a symplectic matrix such that $\Omega_{A B}=-\Omega_{B A}=\left(\Omega^{B A}\right)^{*}, \Omega_{A B} \Omega^{B C}=$ $\delta^{C}{ }_{A}$. The quadratic Casimir operator of $\mathrm{E}_{7(-25)}$ in the basis (3) is given by

$$
\begin{aligned}
\mathcal{C}_{2} & =\frac{1}{6} J^{A}{ }_{B} J^{B}{ }_{A}-\frac{1}{24} \epsilon_{A B C D E F G H} J^{A B C D} J^{E F G H} \\
& =\frac{1}{6} J^{A}{ }_{B} J_{A}^{B}-J^{A B C D}(\epsilon J)_{A B C D}
\end{aligned}
$$

where $(\epsilon J)_{A B C D}=\frac{1}{4 !} \epsilon_{A B C D E F G H} J^{E F G H}$.

The fundamental representation $\mathbf{5 6}$ of $\mathfrak{e}_{7(-25)}$ decomposes as $\mathbf{2 8} \oplus \tilde{\mathbf{2 8}}$ under its $\mathfrak{s u}^{*}(8)$ subalgebra, where $\mathbf{2 8}\left(X^{A B}\right)$ and $\tilde{\mathbf{2 8}}\left(\tilde{X}_{A B}\right)$ are anti-symmetric tensors satisfying the following reality condition

$$
\left(X^{A B}\right)^{\dagger}=X_{A B}=\Omega_{A C} \Omega_{B D} X^{C D}, \quad\left(\tilde{X}_{A B}\right)^{\dagger}=\tilde{X}^{A B}=\Omega^{A C} \Omega^{B D} \tilde{X}_{C D} .
$$

Under the action of $\mathfrak{e}_{7(-25)}$ they transform as

$$
\begin{aligned}
& \delta X^{A B}=\Sigma^{A}{ }_{C} X^{C B}+\Sigma^{B}{ }_{C} X^{A C}-\Sigma^{A B C D} \tilde{X}_{C D} \\
& \delta \tilde{X}_{C D}=-\Sigma^{A}{ }_{C} \tilde{X}_{A D}-\Sigma^{A}{ }_{D} \tilde{X}_{C A}+\Sigma_{C D A B} X^{A B}
\end{aligned}
$$

where $\Sigma^{A}{ }_{C}$ and $\Sigma^{A B C D}=-\left(\Sigma_{A B C D}\right)^{\dagger}$ denote parameters of $\mathrm{SU}^{*}(8)$ transformation and those of the coset generators $\mathrm{E}_{7(-25)} / \mathrm{SU}^{*}(8)$, respectively.

\subsection{Exceptional Lie Algebra $\mathfrak{e}_{8(-24)}$}

Note that $\mathbf{5 6}$ is a real representation of $\mathfrak{e}_{7(-25)}$ just as $\mathbf{2 8}$ and $\tilde{\mathbf{2 8}}$ are real representations of $\mathfrak{s u}^{*}(8)$. Thus in $\mathfrak{s u}^{*}(8)$ covariant basis we can label generators belonging to grade -1 space as $E^{A B}$ and $\tilde{E}_{A B}$ and grade +1 space as $F^{A B}$ and $\tilde{F}_{A B}$. The 5-graded decomposition of $\mathfrak{e}_{8(-24)}$ in $\mathfrak{s u}^{*}(8)$ basis takes the form

$$
\mathfrak{e}_{8(-24)}=E \oplus\left\{E^{A B}, \tilde{E}_{C D}\right\} \oplus\left\{J^{A}{ }_{B}, J^{A B C D} ; \Delta\right\} \oplus\left\{F^{A B}, \tilde{F}_{C D}\right\} \oplus F
$$

The grading is defined by the generator $\Delta$ of $S O(1,1)$

$$
\begin{array}{ll}
{[\Delta, E]=-2 E,} & {[\Delta, F]=+2 F} \\
{\left[\Delta, E^{A B}\right]=-E^{A B},} & {\left[\Delta, F^{A B}\right]=+F^{A B}} \\
{\left[\Delta, \tilde{E}_{C D}\right]=-\tilde{E}_{C D},} & {\left[\Delta, \tilde{F}_{C D}\right]=+\tilde{F}_{C D}}
\end{array}
$$

Positive and negative generators form two separate maximal Heisenberg subalgebras with commutation relations

$$
\left[E^{A B}, \tilde{E}_{C D}\right]=2 \delta_{C D}^{A B} E \quad\left[E, E^{A B}\right]=0 \quad\left[E, \tilde{E}_{A B}\right]=0
$$

and

$$
\left[F^{A B}, \tilde{F}_{C D}\right]=2 \delta_{C D}^{A B} F \quad\left[F, F^{A B}\right]=0 \quad\left[F, \tilde{F}_{A B}\right]=0
$$


However these two Heisenberg subalgebras do not commute with each other (see eqs. (13) below). Generators of $\mathfrak{g}^{ \pm 2}$ are invariant under $\mathfrak{e}_{7(-25)}$

$$
\left[J_{B}^{A}, F\right]=0 \quad\left[J^{A B C D}, F\right]=0 \quad\left[J_{B}^{A}, E\right]=0 \quad\left[J^{A B C D}, E\right]=0
$$

while generators of $\mathfrak{g}^{ \pm 1}$ transform under $\mathfrak{s u}^{*}(8)$ as follows

$$
\begin{aligned}
& {\left[J^{A}{ }_{B}, E^{C D}\right]=\delta^{C}{ }_{B} E^{A D}+\delta^{D}{ }_{B} E^{C A}-\frac{1}{4} \delta^{A}{ }_{B} E^{C D}} \\
& {\left[J^{A}{ }_{B}, F^{C D}\right]=\delta^{C}{ }_{B} F^{A D}+\delta^{D}{ }_{B} F^{C A}-\frac{1}{4} \delta^{A}{ }_{B} F^{C D}} \\
& {\left[J^{A}{ }_{B}, \tilde{E}_{C D}\right]=-\delta^{A}{ }_{C} \tilde{E}_{B D}-\delta^{A}{ }_{D} \tilde{E}_{C B}+\frac{1}{4} \delta^{A}{ }_{B} \tilde{E}_{C D}} \\
& {\left[J^{A}{ }_{B}, \tilde{F}_{C D}\right]=-\delta^{A}{ }_{C} \tilde{F}_{B D}-\delta^{A}{ }_{D} \tilde{F}_{C B}+\frac{1}{4} \delta^{A}{ }_{B} \tilde{F}_{C D}}
\end{aligned}
$$

The remaining commutation relations read as follows

$$
\begin{array}{cl}
{\left[J^{A B C D}, \tilde{E}_{E F}\right]=\delta_{E F}^{[A B} E^{C D]},} & {\left[J^{A B C D}, E^{E F}\right]=-\frac{1}{24} \epsilon^{A B C D E F G H} \tilde{E}_{G H}} \\
{\left[J^{A B C D}, \tilde{F}_{E F}\right]=\delta_{E F}^{[A B} F^{C D]},} & {\left[J^{A B C D}, F^{E F}\right]=-\frac{1}{24} \epsilon^{A B C D E F G H} \tilde{F}_{G H}} \\
{\left[E^{A B}, F^{C D}\right]=-12 J^{A B C D},} & {\left[\tilde{E}_{A B}, F^{C D}\right]=4 \delta_{[A}^{[C} J_{B]}^{D]}+\delta_{A B}^{C D} \Delta} \\
{\left[\tilde{E}_{A B}, \tilde{F}_{C D}\right]=-12(\epsilon J)_{A B C D},} & {\left[E^{A B}, \tilde{F}_{C D}\right]=4 \delta_{[C}^{[A} J_{D]}^{B]}-\delta_{C D}^{A B} \Delta} \\
{\left[E, F^{A B}\right]=-E^{A B},} & {\left[E, \tilde{F}_{A B}\right]=-\tilde{E}_{A B} \quad[E, F]=\Delta} \\
{\left[F, E^{A B}\right]=+F^{A B},} & {\left[F, \tilde{E}_{A B}\right]=+\tilde{F}_{A B}}
\end{array}
$$

Reality properties for generators belonging to grade \pm 1 and \pm 2 are as follows

$$
\begin{aligned}
\left(F^{A B}\right)^{\dagger} & =-\Omega_{A C} \Omega_{B D} F^{C D}, & \left(\tilde{F}_{A B}\right)^{\dagger} & =-\Omega^{A C} \Omega^{B D} \tilde{F}_{C D}, \\
\left(E^{A B}\right)^{\dagger} & =-\Omega_{A C} \Omega_{B D} E^{C D}, & \left(\tilde{E}_{A B}\right)^{\dagger} & =-\Omega^{A C} \Omega^{B D} \tilde{E}_{C D}, \\
E^{\dagger} & =-E, & F^{\dagger} & =-F
\end{aligned}
$$

The quadratic Casimir operator of the above Lie algebra is given by

$$
\begin{aligned}
\mathcal{C}_{2}\left(\mathfrak{e}_{8(-24)}\right) & =\frac{1}{6} J^{A}{ }_{B} J^{B}{ }_{A}-J^{A B C D}(\epsilon J)_{A B C D} \\
& +\frac{1}{12} \Delta^{2}-\frac{1}{6}(F E+E F) \\
& -\frac{1}{12}\left(\tilde{E}_{A B} F^{A B}+F^{A B} \tilde{E}_{A B}-\tilde{F}_{A B} E^{A B}-E^{A B} \tilde{F}_{A B}\right)
\end{aligned}
$$


In order to make manifest the fact that the above Lie algebra is of the real form $\mathfrak{e}_{8(-24)}$ with the maximal compact subalgebra $\mathfrak{e}_{7} \oplus \mathfrak{s u}(2)$ let us write down the compact and noncompact generators explicitly. Under the maximal compact subalgebra $\mathfrak{u s p}(8)$ of $\mathfrak{s u}^{*}(8)$ we have the following decompositions of the adjoint and fundamental representations of $\mathfrak{e}_{8(-24)}$

$$
\begin{aligned}
\mathbf{1 3 3} & =\mathbf{6 3} \oplus \mathbf{7 0}=(\mathbf{3 6} \oplus \mathbf{2 7}) \oplus(\mathbf{1} \oplus \mathbf{2 7} \oplus \mathbf{4 2}) \\
\mathbf{5 6} & =\mathbf{2 8} \oplus \tilde{\mathbf{2 8}}=(\mathbf{1} \oplus \mathbf{2 7}) \oplus(\mathbf{1} \oplus \mathbf{2 7})
\end{aligned}
$$

where $\mathbf{2 7}$ and $\mathbf{4 2}$ correspond to symplectic traceless antisymmetric 2-tensor and 4 -tensor of $\mathfrak{u s p}(8)$ respectively. ${ }^{3}$ Note that the generators in the representations $\mathbf{1} \oplus \mathbf{3 6} \oplus \mathbf{4 2}$ of $\mathfrak{u s p}(8)$ in the decomposition of the adjoint representation of $\mathfrak{e}_{7(-25)}$ form the maximal compact subalgebra $\mathfrak{e}_{6} \oplus \mathfrak{u}(1)$ of $\mathfrak{e}_{7(-25)}$.

Denoting the generators $(T)$ transforming covariantly under the $\mathfrak{u} \mathfrak{s p}(8)$ subalgebra of $\mathfrak{s u}^{*}(8)$ with a check $(\check{T})$ we find that the generators in $\mathbf{3 6} \oplus \mathbf{2 7}$ are given by $\check{G}_{A B}^{( \pm)}=\Omega_{A C} J^{C}{ }_{B} \pm \Omega_{B C} J^{C}$, while generators coming from the decomposition of $\mathbf{7 0}$ with respect to $\mathfrak{u s p}(8)$ are given by

$$
\begin{gathered}
\check{J}^{A B}=J^{A B C D} \Omega_{C D}+\frac{1}{8} \Omega^{A B} \check{J} . \\
\check{J}^{A B C D}:=J^{A B C D}+\frac{3}{2} \Omega^{[A B} \Omega_{E F} J^{C D] E F}+\frac{1}{8} \Omega^{[A B} \Omega^{C D]} \check{J} \\
\check{J}:=\Omega_{E F} \Omega_{G H} J^{E F G H}
\end{gathered}
$$

Thus we find that

$$
J^{A B C D}(\epsilon J)_{A B C D}=\check{J}^{A B C D} \check{J}_{A B C D}-\frac{3}{2} \check{J}^{A B} \check{J}_{A B}+\frac{1}{16} \check{J}^{2}
$$

The decomposition of $\mathbf{5 6}$ of $\mathfrak{e}_{7(-25)}$ into $\mathfrak{u} \mathfrak{s p}(8)$ irreducible components leads to the following generators that transform in the $\mathbf{2 7}$ of $\mathfrak{u s p}(8)$ :

$$
\begin{aligned}
\check{C}_{A B}^{ \pm} & =\tilde{E}_{A B}+F_{A B} \pm\left(\tilde{F}_{A B}-E_{A B}\right) \\
& +\frac{1}{8} \Omega_{A B} \Omega^{C D}\left[\tilde{E}_{C D}+F_{C D} \pm\left(\tilde{F}_{C D}-E_{C D}\right)\right] \\
\check{N}_{A B}^{ \pm} & =\tilde{F}_{A B}+E_{A B} \pm\left(\tilde{E}_{A B}-F_{A B}\right) \\
& +\frac{1}{8} \Omega_{A B} \Omega^{C D}\left[\tilde{F}_{C D}+E_{C D} \pm\left(\tilde{E}_{C D}-F_{C D}\right)\right]
\end{aligned}
$$

and to the following singlets of $\mathfrak{u s p}(8)$ :

$$
\begin{aligned}
& \check{C}^{ \pm}=\Omega^{C D}\left[\tilde{E}_{C D}+F_{C D} \pm\left(\tilde{F}_{C D}-E_{C D}\right)\right] \\
& \check{N}^{ \pm}=\Omega^{C D}\left[\tilde{F}_{C D}+E_{C D} \pm\left(\tilde{E}_{C D}-F_{C D}\right)\right]
\end{aligned}
$$

\footnotetext{
${ }^{3}$ The group $\mathrm{SU}^{*}(8)$ is defined as a subgroup of $\mathrm{SL}(8, \mathbb{C})$ generated by elements $U \in \mathrm{SL}(8, \mathbb{C})$ such that $U^{\dagger} U=1$ and $U \Omega=U^{*} \Omega$. $U^{*}$ is obtained from $U$ by component-wise complex conjugation.
} 
Then the following 133 operators

$$
\check{G}_{A B}^{(+)}, \quad \check{J}^{A B C D}, \quad \check{J}+2(E+F), \quad \check{C}_{A B}^{ \pm}
$$

generate the compact $E_{7}$ subgroup and the operators $\check{C}^{ \pm}$and $2(E+F)-3 \check{J}$ generate the compact $S U(2)$ subgroup. The remaining 112 generators are noncompact:

$$
G_{A B}^{(-)}, \quad \check{J}^{A B}, \quad \Delta, \quad F-E, \quad \check{N}_{A B}^{ \pm}, \quad \check{N}^{ \pm} .
$$

\subsection{The Minimal Unitary Realization of $\mathfrak{e}_{8(-24)}$ in $\mathfrak{s u}^{*}(8)$ Basis}

It was noted earlier that elements of the subspace $\mathfrak{g}^{-2} \oplus \mathfrak{g}^{-1} \subset \mathfrak{e}_{8(-24)}$ form an Heisenberg algebra with 28 "coordinates" and 28 "momenta" with the generator of $\mathfrak{g}^{-2}$ acting as its central charge. As it was done for $\mathfrak{e}_{8(8)}$, we shall realize these Heisenberg algebra generators using canonically conjugate position $\left(X^{A B}\right)$ and momentum $\left(P_{A B}\right)$ operators:

$$
\left[X^{A B}, P_{C D}\right]=i \delta_{C D}^{A B} .
$$

satisfying the following reality properties

$$
\left(X^{A B}\right)^{\dagger}=X_{A B}=\Omega_{A C} \Omega_{B D} X^{C D}, \quad\left(P_{A B}\right)^{\dagger}=P^{A B}=\Omega^{A C} \Omega^{B D} P_{C D}
$$

The commutation relations (20) can also be rewritten in more $\mathfrak{u} \mathfrak{p}(8)$ covariant fashion

$$
\left[X_{A B}, P_{C D}\right]=\frac{i}{2}\left(\Omega_{A C} \Omega_{B D}-\Omega_{B C} \Omega_{A D}\right) .
$$

The generators of $\mathfrak{g}^{-1} \oplus \mathfrak{g}^{-2}$ subalgebra are then realized as

$$
E^{A B}=-i y X^{A B} \quad \tilde{E}_{A B}=-i y P_{A B} \quad E=-\frac{i}{2} y^{2}
$$

where $y$ is an extra coordinate related to central charge. In order to be able to realize $\mathfrak{g}^{+1} \oplus \mathfrak{g}^{+2}$ generators we need to introduce a momentum operator $p$ conjugate to $y$ :

$$
[y, p]=i
$$

The grade zero $\mathfrak{g}^{0}$ generators, realized linearly on operators $X^{A B}$ and $P_{A B}$, take on the form

$$
\begin{aligned}
J^{A}{ }_{B} & =-2 i X^{A C} P_{C B}-\frac{i}{4} \delta^{A}{ }_{B} X^{C D} P_{C D} \\
J^{A B C D} & =-\frac{i}{2} X^{[A B} X^{C D]}-\frac{i}{48} \epsilon^{A B C D E F G H} P_{E F} P_{G H}
\end{aligned}
$$

The dilatation generator $\Delta$ that defines the grading is simply

$$
\Delta=-\frac{i}{2}(p y+y p) \text {. }
$$


Since $\mathfrak{g}^{-1}$ generators are linear and $\mathfrak{g}^{0}$ generators are quadratic polynomials in $X$ and $P$ we expect $\mathfrak{g}^{+1}$ generators to be cubic. Furthermore, $\mathfrak{g}^{+1}=\left[\mathfrak{g}^{+2}, \mathfrak{g}^{-1}\right]$ suggests that $F$ must be a quartic polynomial in $X$ and $P$. Since it is an $\mathfrak{e}_{7(-25)}$ singlet, this quartic must be the quartic invariant of $\mathfrak{e}_{7(-25)}$. Indeed we find

$$
\begin{aligned}
& F=\frac{1}{2 i} p^{2}+\frac{2}{i y^{2}} I_{4}(X, P) \\
& F^{A B}=i p X^{A B}+\frac{2}{y}\left[X^{A B}, I_{4}(X, P)\right] \\
& \tilde{F}_{A B}=i p P_{A B}+\frac{2}{y}\left[P_{A B}, I_{4}(X, P)\right] .
\end{aligned}
$$

The quartic invariant $I_{4}$ coincides with quadratic Casimir of $\mathfrak{e}_{7(-25)}$ modulo an additive constant:

$$
\begin{aligned}
I_{4}(X, P) & =\mathcal{C}_{2}\left(\mathfrak{e}_{7(-25)}\right)+\frac{323}{16}=\frac{547}{16}+ \\
& -\frac{1}{2}\left(X^{A B} P_{B C} X^{C D} P_{D A}+P_{A B} X^{B C} P_{C D} X^{D A}\right) \\
& +\frac{1}{8}\left(X^{A B} P_{A B} X^{C D} P_{C D}+P_{A B} X^{A B} P_{C D} X^{C D}\right) \\
& +\frac{1}{96} \epsilon^{A B C D M N K L} P_{A B} P_{C D} P_{M N} P_{K L} \\
& +\frac{1}{96} \epsilon_{A B C D M N K L} X^{A B} X^{C D} X^{M N} X^{K L}
\end{aligned}
$$

The quadratic Casimir of $\mathfrak{e}_{8(-24)}$ (15) evaluated in the above realization reduces to a c-number as required by the irreducibility. In order to demonstrate that we decompose the quadratic Casimir (15) into three $\mathfrak{e}_{7(-25)}$-invariant pieces

$$
\mathcal{C}_{2}\left(\mathfrak{e}_{8}\right)=\mathcal{C}_{2}\left(\mathfrak{e}_{7}\right)+\mathcal{C}_{2}(\mathfrak{s l}(2, \mathbb{R}))+\mathcal{C}^{\prime}
$$

according to the first, second and the third lines of (15) respectively. From (27) we find that

$$
\mathcal{C}_{2}\left(\mathfrak{e}_{7}\right)=I_{4}-\frac{323}{16} .
$$

Using definitions of $\Delta, E, F$ we obtain

$$
\mathcal{C}_{2}(\mathfrak{s l}(2, \mathbb{R}))=\frac{1}{3} I_{4}-\frac{1}{16}
$$

Using definitions for $\mathfrak{g}^{-1} \oplus \mathfrak{g}^{+1}$ generators we find

$$
\begin{aligned}
3 \mathcal{C}^{\prime} & =7-28 I_{4}-i X^{A B} I_{4} P_{A B}+i P_{A B} I_{4} X^{A B} \\
& =7-28 I_{4}+\left(32 I_{4}-\frac{265}{4}\right)=4 I_{4}-\frac{237}{4}
\end{aligned}
$$

and therefore

$$
\mathcal{C}_{2}\left(\mathfrak{e}_{8}\right)=-40
$$


Since $E_{8}$ does not have any invariant tensors in 58 dimensions (corresponding to 29 position and 29 momentum operators) all higher Casimir operators of $\mathfrak{e}_{8(-24)}$ in the above realization must also reduce to c-numbers as was argued for the case of $\mathfrak{e}_{8(8)}$ in $[6$. By integrating the above Lie algebra one obtains the minimal unitary representation of the group $E_{8(-24)}$ over the Hilbert space of square integrable complex functions in 29 variables.

\subsection{The Minimal Unitary Realization of $\mathfrak{e}_{8(-24)}$ in $\mathfrak{s u}(6,2)$ Basis}

Analysis above was done in $\mathfrak{s u}^{*}(8)$ covariant basis (see footnote on the page 31). Since covariant operators $X^{A B}$ and $P_{A B}$ are position and momenta we refer to this basis as the Schrödinger picture. One can consider an oscillator basis where the natural operators are 28 creation and 28 annihilation operators constructed out of $X$ and $P$ 's. Being complex, we expect them to transform as $\mathbf{2 8} \oplus \overline{\mathbf{2 8}}$ of some non-compact version of $\mathfrak{s u}(8)$ within $\mathfrak{e}_{7(-25)} \subset \mathfrak{g}^{0}$. This algebra turns out to be $\mathfrak{s u}(6,2)$ and the creation and annihilation operators are given as follows

$$
\begin{aligned}
Z^{a b} & =\frac{1}{4} \Gamma^{a b}{ }_{C D}\left(X^{C D}-i P_{C D}\right) \\
\tilde{Z}^{a b} & =\frac{1}{4} \Gamma_{C D}^{a b}\left(X^{C D}+i P_{C D}\right)
\end{aligned}
$$

where transformation coefficient $\Gamma^{a b}{ }_{C D}$ are related to gamma-matrices of

$$
\mathfrak{s o}(6,2) \simeq \mathfrak{s o}^{*}(8) \simeq \mathfrak{s u}^{*}(8) \cap \mathfrak{s u}(6,2)
$$

as spelled out in appendix A. Operators $Z$ and $\tilde{Z}$ satisfy

$$
\left[\tilde{Z}^{a b}, Z^{c d}\right]=\frac{1}{2}\left(\eta^{c a} \eta^{d b}-\eta^{c b} \eta^{d a}\right)
$$

with the following reality conditions

$$
\left(Z^{a b}\right)^{\dagger}=\tilde{Z}^{a b}=\eta^{a c} \eta^{b d} \tilde{Z}_{c d}
$$

where $\eta=\operatorname{Diag}(+,+,+,+,+,+,-,-)$ is used to raise and lower indexes. Generators of $\mathfrak{e}_{7(-25)}$ in this basis take the following form

$$
\begin{aligned}
J^{a}{ }_{b} & =2 Z^{a c} \tilde{Z}_{b c}-\frac{1}{4} \delta_{b}^{a} Z^{c d} \tilde{Z}_{c d} \\
J^{a b c d} & =\frac{1}{2} Z^{[a b} Z^{c d]}-\frac{1}{48} \epsilon^{a b c d e f g h} \tilde{Z}_{e f} \tilde{Z}_{g h}
\end{aligned}
$$

with hermiticity conditions

$$
\left(J_{b}^{a}\right)^{\dagger}=\eta^{a d} \eta_{b c} J_{d}^{c} \quad\left(J^{a b c d}\right)^{\dagger}=-\frac{1}{24} \epsilon_{e f g h}^{a b c d^{e f g h}}
$$


Their commutation relations are

$$
\begin{aligned}
& {\left[J_{b}^{a}, J_{d}^{c}\right]=\delta_{b}^{c} J_{d}^{a}-\delta_{a}^{d} J_{b}^{c}} \\
& {\left[J^{a}{ }_{b}, J^{c d e f}\right]=-4 \delta^{[c}{ }_{b} J^{\text {def }] a}-\frac{1}{2} \delta^{a}{ }_{b} J^{c d e f}} \\
& {\left[J^{a b c d}, J^{e f g h}\right]=\frac{1}{36} \epsilon^{a b c d p[e f g} J_{p}^{h]}}
\end{aligned}
$$

which have the same form as $\mathfrak{s u}^{*}(8)$ covariant eqs. (3). Quadratic Casimir in this basis reads as

$$
\begin{aligned}
\mathcal{C}_{2}\left(\mathfrak{e}_{7}\right)= & \frac{1}{6} J^{a}{ }_{b} J^{b}{ }_{a}+J^{a b c d}(\epsilon J)_{a b c d}=I_{4}(Z, \tilde{Z})-\frac{323}{16}= \\
= & \frac{1}{2}\left(\tilde{Z}_{a b} Z^{b c} \tilde{Z}_{c d} Z^{d a}+Z^{a b} \tilde{Z}_{b c} Z^{c d} \tilde{Z}_{d a}\right) \\
& -\frac{1}{8}\left(\tilde{Z}_{a b} Z^{a b} \tilde{Z}_{c d} Z^{c d}+Z^{a b} \tilde{Z}_{a b} Z^{c d} \tilde{Z}_{c d}\right)+14 \\
& +\frac{1}{96} \epsilon_{a b c d e f g h} Z^{a b} Z^{c d} Z^{e f} Z^{g h}+\frac{1}{96} \epsilon^{a b c d e f g h} \tilde{Z}_{a b} \tilde{Z}_{c d} \tilde{Z}_{e f} \tilde{Z}_{g h}
\end{aligned}
$$

Negative grade generators of $\mathfrak{e}_{8(-24)}$ are then simply

$$
E=\frac{1}{2} y^{2} \quad E^{a b}=y Z^{a b} \quad \tilde{E}_{a b}=y \tilde{Z}_{a b}
$$

Generators in $\mathfrak{g}^{+1}$ can be inferred commuting $\mathfrak{g}^{+2}$ generator

$$
F=\frac{1}{2} p^{2}+2 y^{-2} I_{4}
$$

with generators in $\mathfrak{g}^{-1}$

$$
\begin{aligned}
& F^{a b}=i\left[E^{a b}, F\right]=-p Z^{a b}+2 i y^{-1}\left[Z^{a b}, I_{4}\right] \\
& \tilde{F}_{a b}=i\left[\tilde{E}_{a b}, F\right]=-p \tilde{Z}_{a b}+2 i y^{-1}\left[\tilde{Z}_{a b}, I_{4}\right]
\end{aligned}
$$

or more explicitly

$$
\begin{aligned}
F^{a b}= & -p Z^{a b}-\frac{i}{12} y^{-1} \epsilon^{a b c d e f g h} \tilde{Z}_{c d} \tilde{Z}_{e f} \tilde{Z}_{g h} \\
& +4 i y^{-1} Z^{c[a} \tilde{Z}_{c d} Z^{b] d}+\frac{i}{2} y^{-1}\left(Z^{a b} \tilde{Z}_{c d} Z^{c d}+Z^{c d} \tilde{Z}_{c d} Z^{a b}\right) \\
F_{a b}= & -p \tilde{Z}_{a b}+\frac{i}{12} y^{-1} \epsilon_{a b c d e f g h} Z^{c d} Z^{e f} Z^{g h} \\
& -4 i y^{-1} \tilde{Z}_{c[a} Z^{c d} Z_{b] d}-\frac{i}{2} y^{-1}\left(\tilde{Z}_{a b} Z^{c d} \tilde{Z}_{c d}+\tilde{Z}_{c d} Z^{c d} \tilde{Z}_{a b}\right)
\end{aligned}
$$

We see that commutation relations in this basis closely follow those in $\mathfrak{s u}^{*}(8)$ basis, with modified reality conditions (cf. (36) and (22) as well as (38) with 
(26)). The $S U(6,2)$ covariant commutation relations follow closely those given in section 2.1

$$
\begin{aligned}
& {[E, F]=-\Delta} \\
& {[\Delta, F]=2 F \quad[\Delta, E]=-2 E \quad\left[E, \tilde{F}_{a b}\right]=-i \tilde{E}_{a b}} \\
& {\left[\Delta, F^{a b}\right]=F^{a b} \quad\left[\Delta, E^{a b}\right]=-E^{a b} \quad\left[F, E^{a b}\right]=i F^{a b}} \\
& {\left[\Delta, \tilde{F}_{a b}\right]=\tilde{F}_{a b} \quad\left[\Delta, \tilde{E}_{a b}\right]=-\tilde{E}_{a b} \quad\left[F, \tilde{E}_{a b}\right]=i \tilde{F}_{a b}} \\
& {\left[E, E^{a b}\right]=0 \quad\left[E, \tilde{E}_{a b}\right]=0 \quad\left[\tilde{E}_{a b}, E^{c d}\right]=2 \delta_{a b}^{c d} E} \\
& {\left[F, F^{a b}\right]=0 \quad\left[F, \tilde{F}_{a b}\right]=0 \quad\left[\tilde{F}_{a b}, F^{c d}\right]=2 \delta_{a b}^{c d} F} \\
& {\left[E^{a b}, F^{c d}\right]=-12 i J^{a b c d} \quad\left[\tilde{E}_{a b}, F^{c d}\right]=-4 i \delta_{[a}^{[c} J_{b]}^{d]}-i \delta_{a b}^{c d} \Delta} \\
& {\left[\tilde{E}_{a b}, \tilde{F}_{c d}\right]=12 i(\epsilon J)_{a b c d} \quad\left[E^{a b}, \tilde{F}_{c d}\right]=-4 i \delta_{\left[{ }_{[c} J_{d]}^{b]}+i \delta_{c d}^{a b} \Delta\right.}} \\
& {\left[J^{a}{ }_{b}, E^{c d}\right]=\delta^{c}{ }_{b} E^{a d}+\delta^{d}{ }_{b} E^{c a}-\frac{1}{4} \delta^{a}{ }_{b} E^{c d} \quad\left[J^{a b c d}, \tilde{E}_{e f}\right]=\delta_{e f}^{[a b} E^{c d]}} \\
& {\left[\tilde{E}_{c d}, J^{a}{ }_{b}\right]=\delta^{a}{ }_{c} \tilde{E}_{b d}+\delta^{a}{ }_{d} \tilde{E}_{c b}-\frac{1}{4} \delta^{a}{ }_{b} \tilde{E}_{c d} \quad\left[J^{a b c d}, E^{e f}\right]=\frac{-1}{24} \epsilon^{a b c d e f g h} \tilde{E}_{g h}}
\end{aligned}
$$

The quadratic Casimir of $\mathfrak{e}_{8(-24)}$ in this basis reads as follows

$$
\begin{aligned}
\mathcal{C}_{2} & =\frac{1}{6} J_{b}^{a} J^{b}{ }_{a}+J^{a b c d}(\epsilon J)_{a b c d}+\frac{1}{6}\left(E F+F E+\frac{1}{2} \Delta^{2}\right) \\
& -\frac{i}{12}\left(\tilde{F}_{a b} E^{a b}+E^{a b} \tilde{F}_{a b}-\tilde{E}_{a b} F^{a b}-F^{a b} \tilde{E}_{a b}\right)
\end{aligned}
$$

and reduces to the same c-number as (28).

\section{Truncations of the minimal unitary realiza- tion of $\mathfrak{e}_{8(-24)}$}

Since our realization of $\mathfrak{e}_{8(-24)}$ is non-linear, not every subalgebra of $\mathfrak{e}_{8(-24)}$ can be obtained by a consistent truncation. We consider consistent truncations to subalgebras that are quasi-conformal. Since quasi-conformal algebras admit a 5 -grading

$$
\mathfrak{g}=\mathfrak{g}^{-2} \oplus \mathfrak{g}^{-1} \oplus \mathfrak{g}^{0} \oplus \mathfrak{g}^{+1} \oplus \mathfrak{g}^{+2}
$$

with $\mathfrak{g}^{ \pm 2}$ being one-dimensional, they have an $\mathfrak{s} l(2, \mathbb{R})$ subalgebra generated by elements of $\mathfrak{g}^{ \pm 2}$ and the generator $\Delta$ that determines 5 -grading. However, the quartic invariant $\mathcal{I}_{4}$ will now be that of a subalgebra $\mathfrak{g}^{0}$ of the linearly realized $\mathfrak{e}_{7(-25)}$ within $\mathfrak{e}_{8(-24)}$. Furthermore, this subalgebra must act on the grade \pm 1 subspaces via a symplectic representation. 
Hence, the problem is reduced to enumeration of subalgebras of linearly realized $\mathfrak{e}_{7(-25)}$ admitting a non-degenerate quartic invariant on the symplectic representation. Before giving the explicit truncations below we shall first indicate a partial web of consistent truncations as quasiconformal subalgebras.

Firstly, we can truncate $\mathfrak{e}_{8(-24)}$ down to either $\mathfrak{e}_{7(5)}$ or $\mathfrak{e}_{7(-25)}$, by keeping singlets of either $\mathfrak{s u}(2)$ or $\mathfrak{s u}(1,1)$ within $\mathfrak{s u}(6,2) \subset \mathfrak{e}_{7(-25)}$ correspondingly. Further truncations of $\mathfrak{e}_{7(-25)}$ to rank 6 quasi-conformal algebras can lead to either $\mathfrak{s o}(10,2)$ or $\mathfrak{e}_{6(-14)}$, while truncations of $\mathfrak{e}_{7(5)}$ lead to either $\mathfrak{e}_{6(-14)}$ or $\mathfrak{e}_{6(2)}$ :

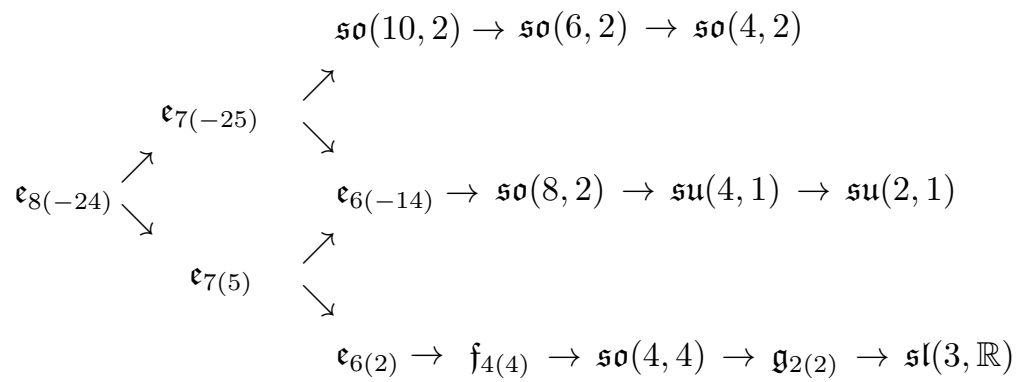

In this paper we shall restrict ourselves to truncations to subalgebras that have rank 3 or higher. The minimal unitary realizations of the rank two Lie groups $\mathrm{G}_{2(2)}$ and $\mathrm{SL}(3, \mathbb{R})$ will be given elsewhere [14. The minimal unitary realization of $\mathrm{SU}(2,1)$ was given in [6].

\subsection{Truncation to the minimal unitary realization of $\mathfrak{e}_{7(-5)}$ as a quasiconformal subalgebra}

In order to truncate the above minimal unitary realization of $\mathfrak{e}_{8(-24)}$ down to its subalgebra $\mathfrak{e}_{7(-5)}$ whose maximal compact subalgebra is $\mathfrak{s o}^{*}(12) \oplus \mathfrak{s u}(2)$ we first observe that $\mathfrak{e}_{7(-5)}$ has the 5 -grading

$$
\mathfrak{e}_{7(-5)}=\begin{array}{ccccccccc}
\mathfrak{g}^{-2} & \oplus & \mathfrak{g}^{-1} & \oplus & \mathfrak{g}^{0} & \oplus & \mathfrak{g}^{+1} & \oplus & \mathfrak{g}^{+2} \\
\mathbf{1} & \oplus & \mathbf{3 2} & \oplus & \left(\mathfrak{s} \mathfrak{o}^{*}(12) \oplus \mathbf{1}\right) & \oplus & \mathbf{3 2} & \oplus & \mathbf{1}
\end{array}
$$

Furthermore, we note that $\mathfrak{e}_{7(-25)}$ has a subalgebra $\mathfrak{s o}^{*}(12) \oplus \mathfrak{s u}(2)$. Hence $\mathfrak{e}_{7(-5)}$ is centralized by an $\mathfrak{s u}(2)$ subalgebra, which can be identified with the one in $\mathfrak{s u}(6) \oplus \mathfrak{s u}(2) \oplus \mathfrak{u}(1) \subset \mathfrak{s u}(6,2) \subset \mathfrak{e}_{7(-25)}$. Under the subalgebra $\mathfrak{s u}(6)$ the adjoint 66 and the spinor representation 32 of $\mathfrak{s o}^{*}(12)$ decompose as follows:

$$
\mathbf{3 2}=\mathbf{1 5} \oplus \mathbf{1} \oplus \overline{\mathbf{1 5}} \oplus \mathbf{1} \quad \text { and } \quad \mathbf{6 6}=\mathbf{3 5} \oplus \mathbf{1 5} \oplus \overline{\mathbf{1 5}} \oplus \mathbf{1} .
$$

This truncation is thus implemented by setting

$$
\begin{aligned}
& \tilde{Z}_{7 b}=0 \text { and } Z^{7 b}=0 \text { where } b \neq 8, \\
& \tilde{Z}_{7 b}=0 \text { and } Z^{8 b}=0 \text { where } b \neq 7,
\end{aligned}
$$

i.e. by restricting to the $\mathfrak{s u}(2)$ singlet sector. 
For the sake of notational convenience, we would retain symbols $Z^{a b}$ and $\tilde{Z}_{a b}$ to denote creation and annihilation operators transforming as $\mathbf{1 5}$ and $\overline{\mathbf{1 5}}$ of $\mathfrak{s u}(6) \subset \mathfrak{s o}^{*}(12)$, where $a$ and $b$ now run from 1 to 6 . Then, generators in $\mathfrak{g}^{-1} \oplus \mathfrak{g}^{-2}$ of $\mathfrak{e}_{7(-5)}$ are given as follows

$$
E=\frac{1}{2} y^{2} \quad E^{a b}=y Z^{a b} \quad E^{+}=y Z^{78} \quad \tilde{E}_{a b}=y \tilde{Z}_{a b} \quad E_{-}=y \tilde{Z}_{78}
$$

The grade zero generators are $\Delta$ and

$$
\begin{aligned}
J_{b}^{a} & =2 Z^{a c} \tilde{Z}_{b c}-\frac{1}{3} \delta^{a}{ }_{b} Z^{c d} \tilde{Z}_{c d} \\
J^{a b} & =\frac{1}{6} Z^{a b} Z^{78}-\frac{1}{48} \epsilon^{a b e f g h} \tilde{Z}_{e f} \tilde{Z}_{g h} \\
\tilde{J}_{a b} & =-\frac{1}{6} \tilde{Z}_{a b} \tilde{Z}_{78}+\frac{1}{48} \epsilon_{a b e f g h} Z^{e f} Z^{g h} \\
H & =-\frac{1}{4}\left(Z^{78} \tilde{Z}_{78}+\tilde{Z}_{78} Z^{78}\right)+\frac{1}{24}\left(Z^{a b} \tilde{Z}_{a b}+\tilde{Z}_{a b} Z^{a b}\right)
\end{aligned}
$$

which form the $\mathfrak{s o}^{*}(12)$ subalgebra. They satisfy the following commutation relations

$$
\begin{aligned}
& \left.\left[J_{b}^{a}, J^{c}{ }_{d}\right]=\delta^{c}{ }_{b} J_{d}^{a}-\delta^{d}{ }_{a} J^{c}{ }_{b} \quad\left[J^{a b}, \tilde{J}_{c d}\right]=\frac{1}{18}\left(2 \delta^{[a}{ }_{[c} J^{b]} d\right]-\delta_{c d}^{a b} H\right) \\
& {\left[J^{a}{ }_{b}, J^{c d}\right]=-2 \delta^{[c}{ }_{b} J^{d] a}-\frac{1}{3} \delta^{a}{ }_{b} J^{c d} \quad\left[H, \tilde{J}_{a b}\right]=-\frac{1}{6} \tilde{J}_{a b}} \\
& {\left[J^{a}{ }_{b}, \tilde{J}_{c d}\right]=2 \delta^{a}{ }_{[c} \tilde{J}_{d] b}+\frac{1}{3} \delta^{a}{ }_{b} \tilde{J}_{c d} \quad\left[H, J^{a b}\right]=\frac{1}{6} J^{a b}}
\end{aligned}
$$

In order to construct positive grade generator we need quadratic Casimir of $\mathfrak{s o}^{*}(12)$ :

$$
\begin{aligned}
\mathcal{C}_{2}\left(\mathfrak{s o}^{*}(12)\right) & =\frac{1}{6} J^{a}{ }_{b} J^{b}{ }_{a}+4 H^{2}+24\left(J^{a b} \tilde{J}_{a b}+\tilde{J}_{a b} J^{a b}\right)=I_{4}-\frac{99}{16}= \\
& =\frac{1}{2}\left(\tilde{Z}_{a b} Z^{b c} \tilde{Z}_{c d} Z^{d a}+Z^{a b} \tilde{Z}_{b c} Z^{c d} \tilde{Z}_{d a}\right) \\
& +\frac{1}{2}\left(Z^{78} \tilde{Z}_{78} Z^{78} \tilde{Z}_{78}+\tilde{Z}_{78} Z^{78} \tilde{Z}_{78} Z^{78}\right)+ \\
& -\frac{1}{8}\left(\tilde{Z}_{a b} Z^{a b} \tilde{Z}_{c d} Z^{c d}+Z^{a b} \tilde{Z}_{a b} Z^{c d} \tilde{Z}_{c d}\right)+4 \\
& -\frac{1}{4}\left(Z^{a b} \tilde{Z}_{a b} Z^{78} \tilde{Z}_{78}+Z^{78} \tilde{Z}_{78} Z^{a b} \tilde{Z}_{a b}\right) \\
& -\frac{1}{4}\left(\tilde{Z}_{a b} Z^{a b} \tilde{Z}_{78} Z^{78}+\tilde{Z}_{78} Z^{78} \tilde{Z}_{a b} Z^{a b}\right) \\
& +\frac{1}{12} \epsilon_{a b c d e f} Z^{a b} Z^{c d} Z^{e f} Z^{78}+\frac{1}{12} \epsilon^{a b c d e f} \tilde{Z}_{a b} \tilde{Z}_{c d} \tilde{Z}_{e f} \tilde{Z}_{78}
\end{aligned}
$$

where the quartic invariant is built out of the spinor representation $\mathbf{3 2}$ of $\mathfrak{s o}^{*}(12)$. Then generators of $\mathfrak{g}^{+1}$ are defined via (38). Commutation relations of $\mathfrak{g}^{0}$ with 
$\mathfrak{g}^{-1}$ read

$$
\begin{aligned}
& {\left[J^{a}{ }_{b}, E^{c d}\right]=-2 \delta^{[c}{ }_{b} E^{d] a}-\frac{1}{3} \delta^{a}{ }_{b} E^{c d} \quad\left[J^{a}{ }_{b}, \tilde{E}_{c d}\right]=2 \delta^{a}{ }_{[c} \tilde{E}_{d] a}+\frac{1}{3} \delta^{a}{ }_{b} \tilde{E}_{c d}} \\
& {\left[J^{a b}, E^{c d}\right]=-\frac{1}{24} \epsilon^{a b c d e f} \tilde{E}_{e f} \quad\left[\tilde{J}_{a b}, E^{c d}\right]=-\frac{1}{6} \delta_{a b}^{c d} \tilde{E}_{78}} \\
& {\left[\tilde{J}_{a b}, \tilde{E}_{c d}\right]=-\frac{1}{24} \epsilon_{a b c d e f} E^{e f} \quad\left[J^{a b}, \tilde{E}_{c d}\right]=-\frac{1}{6} \delta_{c d}^{a b} E^{78}} \\
& {\left[H, E^{a b}\right]=\frac{1}{12} E^{a b} \quad\left[H, \tilde{E}_{a b}\right]=-\frac{1}{12} \tilde{E}_{a b}} \\
& {\left[J^{a b}, E^{78}\right]=0 \quad\left[\tilde{J}_{a b}, E^{78}\right]=-\frac{1}{12} \tilde{E}_{a b} \quad\left[H, E^{78}\right]=-\frac{1}{4} E^{78}} \\
& {\left[\tilde{J}_{a b}, \tilde{E}_{78}\right]=0 \quad\left[J^{a b}, \tilde{E}_{78}\right]=-\frac{1}{12} E^{a b} \quad\left[H, \tilde{E}_{78}\right]=+\frac{1}{4} \tilde{E}_{78}}
\end{aligned}
$$

Commutators of $\mathfrak{s o}^{*}(12)$ generators and the generators belonging to $\mathfrak{g}^{+1}$ subspace are obtained by substituting $E^{a b}$ with $F^{a b}$ and $\tilde{E}_{a b}$ with $\tilde{F}_{a b}$ in equations above. Spaces $\mathfrak{g}^{ \pm 2}$ are $\mathfrak{s o}^{*}(12)$ singlets each. Elements of $\mathfrak{g}^{ \pm 2}$ together with $\Delta$ generate an $\mathfrak{s l}(2, \mathbb{R}) \subset \mathfrak{e}_{7(-5)}$ subalgebra

$$
[E, F]=-\Delta \quad[\Delta, E]=-2 E \quad[\Delta, F]=+2 F .
$$

Generators in $\mathfrak{g}^{-1}$ and $\mathfrak{g}^{+1}$ close into $\mathfrak{g}^{0}$ as follows

$$
\begin{gathered}
{\left[E^{a b}, F^{c d}\right]=-6 i \epsilon^{a b c d e f} \tilde{J}_{e f}} \\
{\left[E^{a b}, \tilde{F}_{c d}\right]=-i \delta_{c d}^{a b}(4 H-\Delta)-4 i \delta_{[c}^{[a} J_{d]}^{b]}} \\
{\left[E^{a b}, F^{78}\right]=-12 i J^{a b} \quad\left[E^{a b}, \tilde{F}_{78}\right]=0} \\
{\left[\tilde{E}_{a b}, F^{c d}\right]=-i \delta_{a b}^{c d}(4 H+\Delta)-4 i \delta_{[a}^{[c} J_{b]}^{d]}} \\
{\left[\tilde{E}_{a b}, \tilde{F}_{c d}\right]=+6 i \epsilon_{a b c d e f} J^{e f}} \\
{\left[\tilde{E}_{a b}, \tilde{F}_{78}\right]=+12 i \tilde{J}_{a b} \quad\left[\tilde{E}_{a b}, F^{78}\right]=0} \\
{\left[E^{78}, F^{a b}\right]=-12 i J^{a b} \quad\left[E^{78}, \tilde{F}_{78}\right]=i\left(\frac{1}{2} \Delta+6 H\right)} \\
{\left[E^{78}, \tilde{F}_{a b}\right]=0 \quad\left[E^{78}, F^{78}\right]=0} \\
{\left[\tilde{E}_{78}, F^{a b}\right]=0 \quad\left[\tilde{E}_{78}, \tilde{F}_{78}\right]=0} \\
{\left[\tilde{E}_{78}, F^{78}\right]=i\left(-\frac{1}{2} \Delta+6 H\right) \quad\left[\tilde{E}_{78}, \tilde{F}_{a b}\right]=+12 i \tilde{J}_{a b}}
\end{gathered}
$$


The resulting realization of $\mathfrak{e}_{7(-5)}$ is that of the minimal unitary representation and the quadratic Casimir of $\mathfrak{e}_{7(-5)}$ reduces to a c-number as required by irreducibility of the minimal unitary representation

$$
\begin{aligned}
\mathcal{C}_{2}\left(\mathfrak{e}_{7(-5)}\right) & =\mathcal{C}_{2}\left(\mathfrak{s o}^{*}(12)\right)+\frac{1}{12} \Delta^{2}+\frac{1}{6}(F E+E F) \\
& -\frac{1}{12}\left(\tilde{E}_{a b} F^{a b}+F^{a b} \tilde{E}_{a b}-\tilde{F}_{a b} E^{a b}-E^{a b} \tilde{F}_{a b}\right) \\
& -\frac{1}{6}\left(\tilde{E}_{78} F^{78}+F^{78} \tilde{E}_{78}-\tilde{F}_{78} E^{78}-E^{78} \tilde{F}_{78}\right) \\
& =\left(I_{4}-\frac{99}{16}\right)+\left(\frac{1}{3} I_{4}-\frac{1}{16}\right)+\left(-\frac{4}{3} I_{4}-\frac{31}{4}\right)=-14
\end{aligned}
$$

\subsection{Truncation to the minimal unitary realization of $\mathfrak{e}_{6(2)}$ as a quasiconformal subalgebra}

Quasi-conformal algebra $\mathfrak{e}_{6(2)}$ with the maximal compact subalgebra $\mathfrak{s u}(6) \oplus$ $\mathfrak{s u}(2)$ has the following 5 -graded decomposition

$$
\mathbf{7 8}=\mathbf{1} \oplus \mathbf{2 0} \oplus(\mathfrak{s u}(3,3) \oplus \Delta) \oplus \mathbf{2 0} \oplus \mathbf{1}
$$

and since $\mathfrak{s u}(3,3) \subset \mathfrak{s o}^{*}(12)$ it can be obtained by the further truncation of $\mathfrak{e}_{7(-5)}$. The maximal compact subalgebra $\mathfrak{s u}(3) \oplus \mathfrak{s u}(3) \oplus \mathfrak{u}(1)$ of $\mathfrak{s u}(3,3)$ is also a subalgebra of $\mathfrak{s u}(6) \subset \mathfrak{s o}^{*}(12)$. This suggests that we split $\mathfrak{s u}(6)$ indices $a=1, \ldots, 6$ into two subsets, $\check{a}=(1,2,3)$ and $\hat{a}=(4,5,6)$, and keep only oscillators which have both types of indices in addition to singlets $\tilde{Z}_{78}$ and $Z^{78}$, i.e. set

$$
Z^{\check{a} \check{c}}=0 \quad \tilde{Z}_{\check{a} \check{c}}=0 \quad Z^{\hat{a} \hat{c}}=0 \quad \tilde{Z}_{\hat{a} \hat{c}}=0
$$

Indeed corresponding $\mathfrak{s u}(3) \oplus \mathfrak{s u}(3) \subset \mathfrak{s u}(3,3)$ branching reads

$$
\mathbf{2 0}=(\mathbf{1}, \mathbf{1}) \oplus(\mathbf{3}, \mathbf{3}) \oplus(\overline{\mathbf{3}}, \overline{\mathbf{3}}) \oplus(\mathbf{1}, \mathbf{1})
$$

This reduction is quite straightforward, and we shall not give here complete commutation relations. All of the formulae of $\mathfrak{e}_{7(-5)}$ carry over to this case provided we set to zero appropriate operators. The quadratic Casimir of $\mathfrak{s u}(3,3)$ that is needed to construct generators of $\mathfrak{g}^{+1} \oplus \mathfrak{g}^{+2}$ reads as follows

$$
\begin{aligned}
& \mathcal{C}_{2}(\mathfrak{s u}(3,3))=\frac{1}{6} J^{\check{a}}{ }_{\breve{c}} J^{\check{c}}{ }_{\breve{a}}+\frac{1}{6} J^{\hat{a}} J_{\hat{c}} J_{\hat{a}}^{\hat{c}}+4 H^{2}+24\left(J^{\check{a} \check{c}} \tilde{J}_{\check{a} \check{c}}+\tilde{J}_{\breve{a} \check{c}} J^{\check{a} \breve{c}}\right) \\
& +24\left(J^{\hat{a} \hat{c}} \tilde{J}_{\hat{a} \hat{c}}+\tilde{J}_{\hat{a} \hat{c}} J^{\hat{a} \hat{c}}\right)=I_{4}-\frac{35}{16}=-\frac{1}{8}\left(\tilde{Z}_{a b} Z^{a b} \tilde{Z}_{c d} Z^{c d}+Z^{a b} \tilde{Z}_{a b} Z^{c d} \tilde{Z}_{c d}\right) \\
& +\frac{1}{2}\left(\tilde{Z}_{a b} Z^{b c} \tilde{Z}_{c d} Z^{d a}+Z^{a b} \tilde{Z}_{b c} Z^{c d} \tilde{Z}_{d a}\right)+\frac{1}{2}\left(Z^{78} \tilde{Z}_{78} Z^{78} \tilde{Z}_{78}+\tilde{Z}_{78} Z^{78} \tilde{Z}_{78} Z^{78}\right) \\
& -\frac{1}{4}\left(Z^{a b} \tilde{Z}_{a b} Z^{78} \tilde{Z}_{78}+Z^{78} \tilde{Z}_{78} Z^{a b} \tilde{Z}_{a b}\right)-\frac{1}{4}\left(\tilde{Z}_{a b} Z^{a b} \tilde{Z}_{78} Z^{78}+\tilde{Z}_{78} Z^{78} \tilde{Z}_{a b} Z^{a b}\right) \\
& +\frac{1}{12} \epsilon_{a b c d e f} Z^{a b} Z^{c d} Z^{e f} Z^{78}+\frac{1}{12} \epsilon^{a b c d e f} \tilde{Z}_{a b} \tilde{Z}_{c d} \tilde{Z}_{e f} \tilde{Z}_{78}+\frac{5}{4}
\end{aligned}
$$


where $Z^{a b}$ and $\tilde{Z}_{a b}$ are as described above, and hence $I_{4}$ is the quadratic invariant of $\mathfrak{s u}(3,3)$ in the representation $\mathbf{2 0}$. The resulting realization of $\mathfrak{e}_{6(2)}$ is again that of the minimal unitary representation. Because some of the oscillators were set equal to zero in the truncation, they do not contribute to the value of the quadratic Casimir of the algebra, the c-number to which it reduces is now different

$$
\mathcal{C}_{2}\left(\mathfrak{e}_{6(2)}\right)=\left(I_{4}-\frac{35}{16}\right)+\left(\frac{1}{3} I_{4}-\frac{1}{16}\right)+\left(-\frac{4}{3} I_{4}-\frac{15}{4}\right)=-6
$$

\subsection{Truncation to the minimal unitary realization of $\mathfrak{e}_{6(-14)}$ as a quasiconformal subalgebra}

Quasiconformal realization of another real form of $\mathfrak{e}_{6}$, namely $\mathfrak{e}_{6(-14)}$ with the maximal compact subalgebra $\mathfrak{s o}(10) \oplus \mathfrak{s o}(2)$, can also be obtained by truncation of $\mathfrak{e}_{7(-5)}$. Its five-graded decomposition reads as follows

$$
\mathfrak{e}_{6(-14)}=\mathbf{1} \oplus \mathbf{2 0} \oplus(\mathfrak{s u}(5,1) \oplus \Delta) \oplus \mathbf{2 0} \oplus \mathbf{1}
$$

In order to implement this truncation we observe the following chain of inclusions

$$
\mathfrak{s u}(5,1) \subset \mathfrak{s o}(10,2) \subset \mathfrak{e}_{7(-25)}
$$

Subalgebra $\mathfrak{s o}(10,2)$ is centralized by $\mathfrak{s u}(1,1)$ while $\mathfrak{s u}(5,1)$ is centralized by $\mathfrak{s u}(2,1)$ within $\mathfrak{e}_{7(-25)}$, suggesting that we only keep oscillators $Z^{a b}$ and $\tilde{Z}_{a b}$ with indexes now running from 1 to 5 as follows from $\mathfrak{u}(5) \subset \mathfrak{s u}(5,1)$ branching of $\mathbf{2 0}=\mathbf{1 0} \oplus \overline{\mathbf{1 0}}$. Then generators of $\mathfrak{s u}(5,1)$ are given as follows

$$
\begin{aligned}
J_{b}^{a} & =2 Z^{a c} \tilde{Z}_{b c}-\frac{2}{5} \delta^{a}{ }_{b} Z^{c d} \tilde{Z}_{c d} \quad H=\frac{1}{24}\left(Z^{a b} \tilde{Z}_{a b}+\tilde{Z}_{a b} Z^{a b}\right) \\
J^{a} & =-\frac{1}{48} \epsilon^{a b c d e} \tilde{Z}_{b c} \tilde{Z}_{d e} \quad \tilde{J}_{a}=+\frac{1}{48} \epsilon_{a b c d e} Z^{b c} Z^{d e}
\end{aligned}
$$

with commutation relations

$$
\begin{array}{rrrr}
{\left[J_{b}^{a}, J^{c}{ }_{d}\right]} & =\delta^{c}{ }_{b} J^{a}{ }_{d}-\delta^{d}{ }_{a} J^{c}{ }_{b} & {\left[J^{a}{ }_{b}, J^{c}\right]=\delta^{c}{ }_{b} J^{a}-\frac{1}{5} \delta^{a}{ }_{b} J^{c}} \\
{\left[J^{a}{ }_{b}, \tilde{J}_{c}\right]} & =-\delta^{a}{ }_{c} \tilde{J}_{b}+\frac{1}{5} \delta^{a}{ }_{b} \tilde{J}_{c} & {\left[H, J^{a}\right]=-\frac{1}{6} J^{a}} & {\left[H, \tilde{J}_{a}\right]=+\frac{1}{6} \tilde{J}_{a}} \\
{\left[J^{a}, \tilde{J}_{b}\right]} & =\frac{1}{144} J^{a}{ }_{b}-\frac{1}{20} \delta^{a}{ }_{b} H & {\left[J^{a}, J^{b}\right]=0} & {\left[\tilde{J}_{a}, \tilde{J}_{b}\right]=0}
\end{array}
$$

resulting in the following Casimir

$$
\begin{aligned}
\mathcal{C}_{2}(\mathfrak{s u}(5,1)) & =\frac{1}{6}{J^{a}}^{a}{J^{b}}^{b}+\frac{36}{5} H^{2}+24\left(J^{a} \tilde{J}_{a}+\tilde{J}_{a} J^{a}\right)=I_{4}-\frac{35}{16} \\
& =\frac{1}{2}\left(\tilde{Z}_{a b} Z^{b c} \tilde{Z}_{c d} Z^{d a}+Z^{a b} \tilde{Z}_{b c} Z^{c d} \tilde{Z}_{d a}\right) \\
& -\frac{1}{8}\left(\tilde{Z}_{a b} Z^{a b} \tilde{Z}_{c d} Z^{c d}+Z^{a b} \tilde{Z}_{a b} Z^{c d} \tilde{Z}_{c d}\right)+\frac{5}{4}
\end{aligned}
$$


Remaining generators of $\mathfrak{e}_{6(-14)}$ and their commutators straightforwardly follow from those of $\mathfrak{e}_{7(-5)}$. We shall only present the c-number to which the quadratic Casimir of $\mathfrak{e}_{6(-14)}$ reduces upon evaluation on the resulting minimal unitary realization

$$
\mathcal{C}_{2}\left(\mathfrak{e}_{6(-14)}\right)=\left(I_{4}-\frac{35}{16}\right)+\left(\frac{1}{3} I_{4}-\frac{1}{16}\right)+\left(-\frac{4}{3} I_{4}-\frac{15}{4}\right)=-6
$$

\subsection{Truncation to the minimal unitary realization of $\mathfrak{f}_{4(4)}$ as a quasiconformal subalgebra}

The realization of the Lie algebra $\mathfrak{e}_{6(2)}$ given above can be further truncated to obtain the minimal unitary realization of the Lie algebra $\mathfrak{f}_{4(4)}$ with the maximal compact subalgebra $\mathfrak{u s p}(6) \oplus \mathfrak{u s p}(2)$. The five graded structure of $\mathfrak{f}_{4(4)}$ as a quasiconformal algebra reads as follows

$$
\mathbf{5 2}=\mathfrak{f}_{4(4)}=\mathbf{1} \oplus \mathbf{1 4} \oplus(\mathfrak{s p}(6, \mathbb{R}) \oplus \Delta) \oplus \mathbf{1 4} \oplus \mathbf{1}
$$

One way to obtain the truncation of $\mathfrak{e}_{6(2)}$ to $\mathfrak{f}_{4(4)}$ is suggested by $\mathfrak{u}(3) \subset \mathfrak{s p}(6, \mathbb{R})$ branching of $\mathbf{1 4}=\mathbf{1} \oplus \mathbf{6} \oplus \overline{\mathbf{6}} \oplus \mathbf{1}$. It amounts to identifying the two $\mathfrak{s u}(3)$ subalgebra of $\mathfrak{s u}(3,3) \subset \mathfrak{e}_{6(2)}$ and discarding the antisymmetric components $Z^{[a b]}$ of $Z^{a b}$.

Let us define the symmetric tensor oscillators $S^{a c}=Z^{(a c)}$ and $\tilde{S}_{a c}=\tilde{Z}_{(a c)}$, $a, b, \ldots=1,2,3$, which correspond to independent oscillators left after the identification. They satisfy the following commutation relations

$$
\left[\tilde{S}_{c d}, S^{a b}\right]=\frac{1}{4}\left(\delta^{a}{ }_{c} \delta^{b}{ }_{d}+\delta^{b}{ }_{c} \delta^{a}{ }_{d}\right)
$$

With these oscillators we build generators of $\mathfrak{s p}(6, \mathbb{R})$ :

$$
\begin{aligned}
J_{b}^{a} & =2 S^{a c} \tilde{S}_{b c}-\frac{2}{3} \delta^{a}{ }_{b} S^{c d} \tilde{S}_{c d} \\
H & =-\frac{1}{4}\left(Z^{78} \tilde{Z}_{78}+\tilde{Z}_{78} Z^{78}\right)+\frac{1}{12}\left(S^{a b} \tilde{S}_{a b}+\tilde{S}_{a b} S^{a b}\right) \\
J^{a b} & =\frac{1}{6} S^{a b} Z^{78}+\frac{1}{12} \epsilon^{a c d} \epsilon^{b e f} \tilde{S}_{c e} \tilde{S}_{d f} \\
\tilde{J}_{a b} & =-\frac{1}{6} \tilde{S}_{a b} \tilde{Z}_{78}-\frac{1}{12} \epsilon_{a c d} \epsilon_{b e f} S^{c e} S^{d f}
\end{aligned}
$$

satisfying the following commutation relations

$$
\begin{aligned}
& {\left[J^{a}{ }_{b}, J^{c d}\right]=\delta^{(c}{ }_{b} J^{d) a}-\frac{1}{3} \delta^{a}{ }_{b} J^{c d}} \\
& {\left[J_{b}^{a}, J^{c}{ }_{d}\right]=\delta^{c}{ }_{b} J^{a}{ }_{d}-\delta^{d}{ }_{a} J^{c}{ }_{b}} \\
& {\left[J^{a}{ }_{b}, \tilde{J}_{c d}\right]=-\delta^{a}{ }_{(c} \tilde{J}_{d) b}+\frac{1}{3} \delta^{a}{ }_{b} \tilde{J}_{c d}} \\
& {\left[H, J^{a b}\right]=-\frac{1}{6} J^{a b}} \\
& {\left[J^{a b}, \tilde{J}_{c d}\right]=\frac{1}{72}\left(\delta_{(c}^{(a} J_{d)}^{b)}-2 \delta_{(c}^{(a}{ }^{b)}{ }_{d)} H\right) \quad\left[H, \tilde{J}_{a b}\right]=\frac{1}{6} \tilde{J}_{a b}}
\end{aligned}
$$


The quadratic Casimir of $\mathfrak{s p}(6, \mathbb{R})$ is then given by

$$
\begin{aligned}
\mathcal{C}_{2}(\mathfrak{s p}(6, \mathbb{R}))= & \frac{1}{3} J^{a}{ }_{b} J^{b}{ }_{a}+4 H^{2}+24\left(J^{a b} \tilde{J}_{a b}+\tilde{J}_{a b} J^{a b}\right)=I_{4}-\frac{15}{16} \\
& =\left(\tilde{S}_{a b} S^{b c} \tilde{S}_{c d} S^{d a}+S^{a b} \tilde{S}_{b c} S^{c d} \tilde{S}_{d a}\right) \\
& +\frac{1}{2}\left(Z^{78} \tilde{Z}_{78} Z^{78} \tilde{Z}_{78}+\tilde{Z}_{78} Z^{78} \tilde{Z}_{78} Z^{78}\right)+ \\
& -\frac{1}{2}\left(\tilde{S}_{a b} S^{a b} \tilde{S}_{c d} S^{c d}+S^{a b} \tilde{S}_{a b} S^{c d} \tilde{S}_{c d}\right)+\frac{7}{16} \\
& -\frac{1}{2}\left(S^{a b} \tilde{S}_{a b} Z^{78} \tilde{Z}_{78}+Z^{78} \tilde{Z}_{78} S^{a b} \tilde{S}_{a b}\right) \\
& -\frac{1}{2}\left(\tilde{S}_{a b} S^{a b} \tilde{Z}_{78} Z^{78}+\tilde{Z}_{78} Z^{78} \tilde{S}_{a b} S^{a b}\right) \\
& -\frac{2}{3} \epsilon_{a b c} \epsilon_{d e f} S^{a d} S^{b e} S^{c f} Z^{78}-\frac{2}{3} \epsilon^{a b c} \epsilon^{\operatorname{def}} \tilde{S}_{a d} \tilde{S}_{b e} \tilde{S}_{c f} \tilde{Z}_{78}
\end{aligned}
$$

Negative grade generators are defined as

$$
E=\frac{1}{2} y \quad E^{a b}=y S^{a b} \quad E^{+}=y Z^{78} \quad \tilde{E}_{a b}=y \tilde{S}_{a b} \quad E_{-}=y \tilde{Z}_{78}
$$

They satisfy commutation relations, different from those of negative grade generators of $\mathfrak{e}_{7(-5)}$

$$
\left[\tilde{E}_{a b}, E^{c d}\right]=\delta_{\left({ }^{(c} \delta^{b)}\right)} E
$$

reflecting that $S^{a b}$ and $\tilde{S}_{a b}$ are now symmetric tensor oscillators. Positive grade generators, and their commutator, are given by the following equations

$$
\begin{aligned}
F & =\frac{1}{2} p^{2}+2 i y^{-2} I_{4} \\
F^{a b} & =-p S^{a b}+2 i y^{-1}\left[S^{a b}, I_{4}\right] \quad\left[\tilde{F}_{a b}, F^{c d}\right]=\delta^{(c}{ }_{(b} \delta^{b)}{ }_{d)} F \\
\tilde{F}_{a b} & =-p \tilde{S}_{a b}+2 i y^{-1}\left[\tilde{S}_{a b}, I_{4}\right]
\end{aligned}
$$

Quadratic Casimir of the resulting minimal unitary realization of $\mathfrak{f}_{4(4)}$

$$
\begin{aligned}
\mathcal{C}_{2}\left(\mathfrak{f}_{4(4)}\right) & =\mathcal{C}_{2}(\mathfrak{s p}(6, \mathbb{R}))+\frac{1}{12} \Delta^{2}+\frac{1}{6}(F E+E F) \\
& +i\left(\tilde{E}_{a b} F^{a b}+F^{a b} \tilde{E}_{a b}-\tilde{F}_{a b} E^{a b}-E^{a b} \tilde{F}_{a b}\right) \\
& -\frac{i}{6}\left(\tilde{E}_{78} F^{78}+F^{78} \tilde{E}_{78}-\tilde{F}_{78} E^{78}-E^{78} \tilde{F}_{78}\right)
\end{aligned}
$$

reduces to a c-number

$$
\mathcal{C}_{2}\left(\mathfrak{f}_{4(4)}\right)=\left(I_{4}-\frac{15}{16}\right)+\left(\frac{1}{3} I_{4}-\frac{1}{16}\right)+\left(-\frac{4}{3} I_{4}-\frac{9}{4}\right)=-\frac{13}{4}
$$

in agreement with parent algebras and as required by irreducibility. 


\subsection{Truncation to the minimal unitary realization of $\mathfrak{s o}(4,4)$ as a quasiconformal subalgebra}

We further truncate $\mathfrak{f}_{4(4)}$ to obtain the minimal unitary realization of $\mathfrak{s o}(4,4)$ which has the following 5 -graded decomposition

$$
\mathbf{2 8}=\mathbf{1} \oplus(\mathbf{2}, \mathbf{2}, \mathbf{2}) \oplus(\mathfrak{s p}(2, \mathbb{R}) \oplus \mathfrak{s p}(2, \mathbb{R}) \oplus \mathfrak{s p}(2, \mathbb{R}) \oplus \Delta) \oplus(\mathbf{2}, \mathbf{2}, \mathbf{2}) \oplus \mathbf{1}
$$

This truncation is achieved by restricting $S^{a b}$ and $\tilde{S}_{a b}$ operators to their diagonal components

$$
S^{a b}=\delta^{a b} S^{a} \quad \tilde{S}_{a b}=\delta_{a b} \tilde{S}_{a} \quad\left[\tilde{S}_{a}, S^{b}\right]=\frac{1}{2} \delta^{b}{ }_{a}
$$

where $a, b, . .=1,2,3$, and discarding the off-diagonal oscillators. Three copies of $\mathfrak{s p}(2, \mathbb{R})$ are generated by

$$
\begin{aligned}
& J_{-}^{a}=-\frac{1}{6} \tilde{S}_{a} \tilde{Z}_{78}-\frac{1}{12} \epsilon_{a b c} S^{b} S^{c} \\
& J_{+}^{a}=\frac{1}{6} S^{a} Z^{78}+\frac{1}{12} \epsilon^{a b c} \tilde{S}_{b} \tilde{S}_{c}
\end{aligned} \quad J_{0}^{a}=Z^{78} \tilde{Z}_{78}+\sum_{b=1}^{3}\left(2 \delta^{a b}-1\right) S^{b} \tilde{S}_{b}
$$

The quadratic Casimir of $\mathfrak{s p}(2, \mathbb{R}) \oplus \mathfrak{s p}(2, \mathbb{R}) \oplus \mathfrak{s p}(2, \mathbb{R})$ then reads

$$
\begin{aligned}
\mathcal{C}_{2} & (\mathfrak{s p}(2, \mathbb{R}) \oplus \mathfrak{s p}(2, \mathbb{R}) \oplus \mathfrak{s p}(2, \mathbb{R}))=\sum_{a=1}^{3}\left[\frac{1}{3} J_{0}^{a} J_{0}^{a}+24\left(J_{-}^{a} J_{+}^{a}+J_{+}^{a} J_{-}^{a}\right)\right] \\
& =\sum_{a=1}^{3}\left(\left(\tilde{S}_{a} S^{a}\right)^{2}+\left(S^{a} \tilde{S}_{a}\right)^{2}\right)+\left(Z^{78} \tilde{Z}_{78}\right)^{2}+\left(\tilde{Z}_{78} Z^{78}\right)^{2} \\
& -\frac{1}{2}\left(\sum_{a=1}^{3} S^{a} \tilde{S}_{a}+Z^{78} \tilde{Z}_{78}\right)^{2}-\frac{1}{2}\left(\sum_{a=1}^{3} \tilde{S}_{a} S^{a}+\tilde{Z}_{78} Z^{78}\right)^{2} \\
& -4 S^{1} S^{2} S^{3} Z^{78}-4 \tilde{S}_{1} \tilde{S}_{2} \tilde{S}_{3} \tilde{Z}_{78}=I_{4}-\frac{3}{16}
\end{aligned}
$$

The commutation relations of the generators in $\mathfrak{g}^{0}$ are

$$
\left[J_{0}^{a}, J_{ \pm}^{b}\right]= \pm \delta^{a b} J_{ \pm}^{a} \quad\left[J_{+}^{a}, J_{-}^{b}\right]=\frac{1}{72} \delta^{a b} J_{0}^{a}
$$

Negative grade generators are

$$
E=\frac{1}{2} y^{2} \quad E^{a}=y S^{a} \quad \tilde{E}_{a}=y \tilde{S}_{a} \quad E^{78}=y Z^{78} \quad \tilde{E}_{78}=y \tilde{Z}_{78}
$$

and positive grade generators are

$$
\begin{aligned}
F^{a} & =-p S^{a}+2 i y^{-1}\left[S^{a}, I_{4}\right] \\
F^{78} & =-p Z^{78}+2 i y^{-1}\left[Z^{78}, I_{4}\right] \\
\tilde{F}_{a} & =-p \tilde{S}_{a}+2 i y^{-1}\left[\tilde{S}_{a}, I_{4}\right] \\
\tilde{F}_{78} & =-p \tilde{Z}_{78}+2 i y^{-1}\left[\tilde{Z}_{78}, I_{4}\right]
\end{aligned}
$$


The quadratic Casimir of $\mathfrak{s o}(4,4)$

$$
\begin{aligned}
\mathcal{C}_{2}(\mathfrak{s o}(4,4)) & =\mathcal{C}_{2}\left(\mathfrak{g}^{0}\right)+\frac{1}{12} \Delta^{2}+\frac{1}{6}(F E+E F) \\
& +\frac{i}{6}\left(E^{a} \tilde{F}_{a}+\tilde{F}_{a} E^{a}-\tilde{E}_{a} F^{a}-F^{a} \tilde{E}_{a}\right) \\
& +\frac{i}{6}\left(E^{78} \tilde{F}_{78}+\tilde{F}_{78} E^{78}-F^{78} \tilde{E}_{78}-\tilde{E}_{78} F^{78}\right)
\end{aligned}
$$

reduces to c-number as before

$$
\mathcal{C}_{2}(\mathfrak{s o}(4,4))=\left(I_{4}-\frac{3}{16}\right)+\left(\frac{1}{3} I_{4}-\frac{1}{16}\right)+\left(-\frac{4}{3} I_{4}-\frac{13}{12}\right)=-\frac{4}{3}
$$

\section{Truncation to the minimal unitary realization of $\mathfrak{e}_{7(-25)}$ as a quasiconformal subalgebra}

The group $\mathrm{E}_{7(-25)}$ has the maximal compact subgroup $E_{6} \times U(1)$ and arises as the $U$-duality group of exceptional $\mathcal{N}=2$ Maxwell-Einstein supergravity in $d=$ 4 whose scalar manifold is $\mathrm{E}_{7(-25)} /\left(E_{6} \times U(1)\right)$. Its action on the 27 complex scalar fields can be represented as a generalized conformal group [10, 16, 8]. As a generalized conformal group its Lie algebra has a natural 3-graded structure

$$
\mathfrak{e}_{7(-25)}=\overline{27} \oplus\left(\mathfrak{e}_{6(-26)} \oplus \mathfrak{s o}(1,1)\right) \oplus 27
$$

The quasiconformal realization of $E_{8(-24)}$ can be truncated to the conformal realization of $E_{7(-25)}$ in essentially two different ways.

In this section we will however consider a different truncation of $E_{8(-24)}$ such that the resulting realization of $E_{7(-25)}$ is quasiconformal corresponding to its minimal unitary representation.

Just as the subalgebra $\mathfrak{e}_{7(-5)}$ is normalized by $\mathfrak{s u}(2) \subset \mathfrak{s u}(6,2) \subset \mathfrak{g}^{0}=$ $\mathfrak{e}_{7(-25)}$, the subalgebra $\mathfrak{e}_{7(-25)}$ is normalized by $\mathfrak{s u}(1,1) \subset \mathfrak{s u}(6,2) \subset \mathfrak{g}^{0} \mathfrak{e}_{7(-25)}$ within $\mathfrak{e}_{8(-24)}$. Similarly to $\mathfrak{e}_{7(-5)}$ we obtain

$$
\mathfrak{e}_{7(-25)}=\mathbf{1 3 3}=\mathbf{1} \oplus \mathbf{3 2} \oplus(\mathfrak{s o}(10,2) \oplus \Delta) \oplus \mathbf{3 2} \oplus \mathbf{1}
$$

We identify the $\mathfrak{s u}(1,1)$ in question with the one generated by $J^{6}{ }_{7}, J^{7}{ }_{6}$ and $J^{6}{ }_{6}-J^{7} 7$ generators of $\mathfrak{s u}(6,2) \subset \mathfrak{e}_{7(-25)} \subset \mathfrak{e}_{8(-24)}$. The truncation will then amount to setting $Z^{a 6}=Z^{6 a}=0$ where $a \neq 7$, as well as $Z^{a 7}=Z^{7 a}=0$ for $a \neq 6$. Let us relable coefficients and introduce $\dot{a}=1, \ldots, 5,8$. Then $\mathfrak{s u}(5,1)$ is generated by

$$
J^{\dot{a}}{ }_{\dot{b}}=2 Z^{\dot{a} \dot{c}} \tilde{Z}_{\dot{b} \dot{c}}-\frac{1}{3} \delta^{\dot{a}} Z^{\dot{d} \dot{c}} \tilde{Z}_{\dot{d} \dot{c}}
$$


The other generators of $\mathfrak{s o}(10,2)$ are then given as follows

$$
\begin{aligned}
U & =\frac{3}{2}\left(Z^{67} \tilde{Z}_{67}+\tilde{Z}_{67} Z^{67}\right)-\frac{1}{4}\left(Z^{\dot{a} \dot{b}} \tilde{Z}_{\dot{a} \dot{b}}+\tilde{Z}_{\dot{a} \dot{b}} Z^{\dot{a} \dot{b}}\right) \\
J_{\dot{a} \dot{b}}^{-} & =-\frac{1}{6} \tilde{Z}_{\dot{a} \dot{b}} \tilde{Z}_{67}+\frac{1}{48} \epsilon_{\dot{a} \dot{b} \dot{c} \dot{d} \dot{e} \dot{f}} Z^{\dot{c} \dot{d}} Z^{\dot{e} \dot{f}} \\
J_{+}^{\dot{a} \dot{b}} & =\frac{1}{6} Z^{\dot{a} \dot{b}} Z^{67}-\frac{1}{48} \epsilon^{\dot{a} \dot{b} \dot{c} \dot{d} \dot{e} \dot{f}} \tilde{Z}_{\dot{c} \dot{d}} \tilde{Z}_{\dot{e} \dot{f}}
\end{aligned}
$$

satisfying the following hermiticity condition

$$
\left(J^{\dot{a}}\right)^{\dagger}=\eta^{\dot{a} \dot{c}} \eta_{\dot{b} \dot{d}} J_{\dot{c}}^{\dot{d}} \quad U^{\dagger}=U \quad\left(J_{\dot{a} \dot{b}}^{-}\right)^{\dagger}=J_{+}^{\dot{c} \dot{d}} \eta_{\dot{a} \dot{c}} \eta_{\dot{b} \dot{d}}
$$

where $\eta_{\dot{a} \dot{b}}=\operatorname{diag}(+1,+1,+1,+1,+1,-1)$; and the commutation relations read as follows

$$
\begin{aligned}
{\left[J^{\dot{a}}{ }_{b}, J^{\dot{c}}{ }_{\dot{d}}\right] } & =\delta^{\dot{c}}{ }_{\dot{b}} J^{\dot{a}}{ }_{\dot{d}}-\delta^{\dot{a}}{ }_{\dot{d}} J^{\dot{c}}{ }_{\dot{b}} \\
{\left[J^{\dot{a}}{ }_{\dot{b}}, J_{+}^{\dot{c} \dot{d}}\right] } & =\delta^{\dot{c}}{ }_{\dot{b}} J_{+}^{\dot{a} \dot{d}}+\delta^{\dot{d}}{ }_{\dot{b}} J_{+}^{\dot{c} \dot{a}}-\frac{1}{3} \delta^{\dot{a}}{ }_{\dot{b}} J_{+}^{\dot{c} \dot{d}} \\
{\left[J^{\dot{a}}{ }_{\dot{b}}, J_{\dot{c} \dot{d}}^{-}\right] } & =-\delta^{\dot{a}}{ }_{\dot{c}} J_{\dot{b} \dot{d}}^{-}-\delta^{\dot{a}}{ }_{\dot{d}} J_{\dot{c} \dot{b}}^{-}+\frac{1}{3} \delta^{\dot{a}}{ }_{\dot{b}} J_{\dot{c} \dot{d}}^{-} \\
{\left[U, J_{\dot{c} \dot{d}}^{-}\right] } & =-J_{\dot{c} \dot{d}}^{-} \quad\left[U, J_{+}^{\dot{c} \dot{d}}\right]=+J_{+}^{\dot{c} \dot{d}} \quad\left[U, J^{\dot{c}}{ }_{\dot{d}}\right]=0
\end{aligned}
$$

The quadratic Casimir of the algebra reads

$$
\mathcal{C}_{2}(\mathfrak{s o}(10,2))=\frac{1}{6} J^{\dot{a}}{ }_{\dot{b}} J^{\dot{b}}{ }_{\dot{a}}+\frac{1}{9} U^{2}+12\left(J_{+}^{\dot{a} \dot{b}} J_{\dot{a} \dot{b}}^{-}+J_{\dot{a} \dot{b}}^{-} J_{+}^{\dot{a} \dot{b}}\right)=I_{4}-\frac{99}{16}
$$

Definition of the grade \pm 1 generators goes along the same lines as for $\mathfrak{e}_{7(-5)}$ so we omit them here. Let us only note that the quadratic Casimir of the minimal unitary realization of $\mathfrak{e}_{7(-25)}$ takes on the same value as that of $\mathfrak{e}_{7(-5)}$ and equals to -14 .

\subsection{Truncations to minimal realizations of $S O(2 p, 2)(p=$ $2,3,4,5)$ as quasiconformal subgroups}

The minimal unitary realization of $\mathrm{E}_{7(-25)}$ can be further truncated to obtain the minimal unitary realizations of subgroups of the from $S O(2 p, 2)$. To this end consider the 5-grading of $\mathrm{E}_{7(-25)}$

$$
\mathbf{1 3 3}=\mathbf{1} \oplus \mathbf{3 2} \oplus(\mathfrak{s o}(10,2) \oplus \Delta) \oplus \mathbf{3 2} \oplus \mathbf{1}
$$

The Lie algebra $\mathfrak{s o}(10,2)$ has a subalgebra

$$
\mathfrak{s o}(2,2) \oplus \mathfrak{s o}(8)=\mathfrak{s u}(1,1)_{L} \oplus \mathfrak{s u}(1,1)_{R} \oplus \mathfrak{s o}(8)
$$

under which its spinor representation 32 decomposes $\mathrm{as}^{4}$

$$
32=\left(\mathbf{2}, \mathbf{1}, \mathbf{8}_{c}\right) \oplus\left(\mathbf{1}, \mathbf{2}, \mathbf{8}_{s}\right)
$$

\footnotetext{
${ }^{4}$ In the decomposition of the other spinor representation $\mathbf{3 2}{ }^{\prime}$ the $\boldsymbol{8}_{c}$ and $\boldsymbol{8}_{s}$ are interchanged.
} 
By restricting ourselves to $\mathrm{SU}(1,1)_{R}$ singlets we obtain the minimal unitary realization of $\mathrm{SO}(10,2)$ subgroup of $\mathrm{E}_{7(-25)}$

$$
\mathfrak{s o}(10,2)=\mathbf{1} \oplus\left(\mathbf{2}, \mathbf{1}, \mathbf{8}_{c}\right) \oplus\left(\mathfrak{s u}(1,1)_{L} \oplus \mathfrak{s o}(8) \oplus \Delta\right) \oplus\left(\mathbf{2}, \mathbf{1}, \mathbf{8}_{c}\right) \oplus \mathbf{1}
$$

By restricting to the $\mathrm{SU}(1,1)_{L}$ singlets one obtains a $\mathrm{SO}(8)$ triality rotated realization of $\mathrm{SO}(10,2)$

$$
\mathfrak{s o}(10,2)=\mathbf{1} \oplus\left(\mathbf{1}, \mathbf{2}, \mathbf{8}_{s}\right) \oplus\left(\mathfrak{s u}(1,1)_{R} \oplus \mathfrak{s o}(8) \oplus \Delta\right) \oplus\left(\mathbf{1}, \mathbf{2}, \mathbf{8}_{s}\right) \oplus \mathbf{1}
$$

We recall the decomposition of the three 8 dimensional irreps of $\mathrm{SO}(8)$ with respect to its $\mathrm{SU}(4) \times \mathrm{U}(1)$ subgroup 13

$$
\begin{array}{r}
\boldsymbol{8}_{v}=\mathbf{4}^{(1)}+\overline{\mathbf{4}}^{(-1)} \\
\mathbf{8}_{s}=\mathbf{1}^{(2)}+\boldsymbol{6}^{(0)}+\mathbf{1}^{(-2)} \\
\mathbf{8}_{c}=\mathbf{4}^{(-1)}+\overline{\mathbf{4}}^{(1)}
\end{array}
$$

Thus by splitting the indices $\dot{a}, \dot{b}, .$. as

$$
\dot{a}=(\mu, x) \quad \mu=1,2,3,4 \quad x=5,8
$$

and identifying the generators of the $\mathrm{U}(4)$ subgroup of $\mathrm{SO}(8)$ with $J_{\nu}^{\mu}$ and the $\mathrm{SU}(1,1)_{L}$ generators with $\left(J^{x}{ }_{y}-\frac{1}{2} \delta^{x}{ }_{y}{ }_{z}^{z}\right)$ we find the following decompositions of the oscillators with respect to the $\mathrm{SU}(1,1)_{L} \times \mathrm{SU}(1,1)_{R} \times \mathrm{SO}(8)$

$$
\begin{aligned}
\left(\tilde{Z}_{\mu x}, Z^{\nu, y}\right) & =\left(\mathbf{2}, \mathbf{1}, \mathbf{8}_{c}\right) \\
\left(\tilde{Z}_{\mu \nu}, Z^{\mu \nu}, \tilde{Z}_{x y}, Z^{x y}, \tilde{Z}_{67}, Z^{67}\right) & =\left(\mathbf{1}, \mathbf{2}, \mathbf{8}_{s}\right)
\end{aligned}
$$

By setting either set of these 16 operators equal to zero we get a consistent truncation of the minimal unitary realization of $\mathrm{E}_{7(-25)}$ to one of its $\mathrm{SO}(10,2)$ quasiconformal subgroups. Here we give the realization obtained by setting the operators in the $\left(\mathbf{1}, \mathbf{2}, \mathbf{8}_{s}\right)$ representation equal to zero.

The $\mathfrak{s o}(8)$ generators in the grade zero subspace of $\mathfrak{s o}(10,2)$ are given by

$$
\mathfrak{s o}(8)=\left(J_{\mu \nu}^{-}, J_{\nu}^{\mu}, J_{+}^{\mu \nu}\right)
$$

where

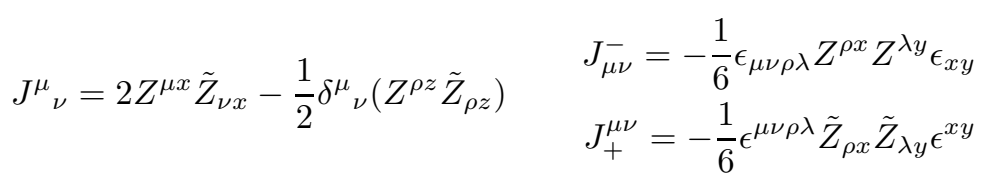

The quadratic Casimir of the grade zero subalgebra $\mathfrak{s o}(8) \oplus \mathfrak{s u}(1,1)$ takes the form

$$
C_{2}(\mathfrak{s o}(8) \oplus \mathfrak{s u}(1,1))=\frac{1}{6}\left(J_{\nu}^{\mu} J_{\mu}^{\nu}+{J^{x}}_{y} J^{y}{ }_{x}+U^{2}\right)+12\left(J_{+}^{\mu \nu} J_{\mu \nu}^{-}+J_{\mu \nu}^{-} J_{+}^{\mu \nu}\right)
$$


where

$$
J^{x}=2 Z^{\mu x} \tilde{Z}_{\mu y}-\delta_{y}^{x}\left(Z^{\mu z} \tilde{Z}_{\mu z}\right)
$$

and

$$
U=-\frac{1}{2}\left(Z^{\mu x} \tilde{Z}_{\mu x}+\tilde{Z}_{\mu x} Z^{\mu x}\right)
$$

The grade -1 generators of $\mathrm{SO}(10,2)$ are simply given by

$$
E^{\mu x}=y Z^{\mu x} \quad \tilde{E}_{\mu x}=y \tilde{Z}_{\mu x}
$$

and those of grade +1 are given by

$$
F^{\mu x}=-p Z^{\mu x}+\frac{2 i}{y}\left[Z^{\mu x}, I_{4}\right] \quad \tilde{F}_{\mu x}=-p \tilde{Z}_{\mu x}+\frac{2 i}{y}\left[\tilde{Z}_{\mu x}, I_{4}\right]
$$

where grade +2 generator $F$ is given by

$$
F=\frac{1}{2} p^{2}+\frac{2}{y^{2}} I_{4} \quad I_{4}=\mathcal{C}_{2}(\mathfrak{s o}(8) \oplus \mathfrak{s u}(1,1))+\frac{73}{48}
$$

The algebra's quadratic Casimir equals

$$
\mathcal{C}_{2}(\mathfrak{s o}(10,2))=-4
$$

To obtain the truncations of the above realization of $\mathfrak{s o}(10,2)$ to the minimal unitary realizations of $\mathfrak{s o}(2 p+2,2)$ we need only restrict the indices $\mu, \nu, \ldots$ of non-vanishing oscillators to run over $\mu, \nu, . .=1, . ., p$, where $p=1,2,3$.

\section{Discussion}

We note that the quadratic Casimir of quasi-conformal algebras evaluated on the minimal realization is related to the algebra's dual Coxeter number $g^{\vee}$

$$
\mathcal{C}_{2}=-\frac{1}{108} g^{\vee}\left(5 g^{\vee}-6\right)
$$

for cases where $g^{0}$ is simple. This is a reflection of the fact that quasi-conformal algebras can be constructed in a unified manner. In a forthcoming paper we will give such a unified approach to the construction of the minimal unitary representations of all simple noncompact groups [14.

Here we would like to stress that the minimal unitary realizations given above, in [6] as well as in the unified approach [14 correspond to quantization of the geometric action of the respective noncompact group as a quasiconformal group as defined and studied for the exceptional groups in [8. A quasiconformal group $G$ leaves invariant a generalized light-cone with respect to a distance function defined in terms of the quartic invariant of its subgroup $H$ which is the normalizer of the $\mathrm{SL}(2, \mathbb{R})$ subgroup generated by grade \pm 2 elements of its Lie algebra $\mathfrak{g}^{5}$. The realization of this $\operatorname{SL}(2, \mathbb{R})$ subgroup inside the minimal

\footnotetext{
${ }^{5}$ For some of the classical noncompact groups the corresponding quartic invariant may be degenerate 14 .
} 
unitary realization is precisely of the form that arises in conformal quantum mechanics [15] as was stressed in [6]. The quartic invariant of the subgroup $H$ plays the role of coupling constant in the corresponding conformal quantum mechanics.

\section{Appendix}

\section{A Going from $\mathfrak{s u}^{*}(8)$ to $\mathfrak{s u}(6,2)$ basis}

Recall that position and momentum operators $X^{A B}$ and $P_{A B}$ transform as $\mathbf{2 8}$ and $\tilde{\mathbf{2 8}}$ under $\mathfrak{s u}^{*}(8)$. To build annihilation and creation operators we need to take complex linear combinations of the form $X^{A B} \pm i P_{A B}$, which transform covariantly under $\mathfrak{s o}^{*}(8)$ subalgebra of $\mathfrak{s u}^{*}(8)$. We expect resulting creation and annihilation operators to transform as $\mathbf{2 8}$ and $\overline{\mathbf{2 8}}$ of some non-compact form of $\mathfrak{s u}(8)^{6}$. The isomorphism $\mathfrak{s o}^{*}(8) \simeq \mathfrak{s o}(6,2)$ suggests that this non-compact form should be $\mathfrak{s u}(6,2)$ as we shall establish.

In order to elucidate the role of triality of $\mathfrak{s o}(8)$ we recall that adjoint representation of compact $\mathfrak{e}_{7}$ decomposes into four representations of $\mathfrak{s o}(8)$ :

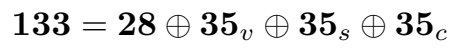

where three $\mathbf{3 5}$ correspond to symmetric traceless tensor in $\mathbf{8}_{v} \otimes \mathbf{8}_{v}, \boldsymbol{8}_{s} \otimes \mathbf{8}_{s}$ and $\boldsymbol{8}_{c} \otimes \mathbf{8}_{c}$ respectively, with $\mathbf{8}_{v}, \mathbf{8}_{s}$ and $\boldsymbol{8}_{c}$ being three inequivalent eight dimensional representations of $\mathfrak{s o}(8)$. Triality of $\mathfrak{s o}(8)$ then maps 35 representations into one another. Observe also, that $\mathbf{2 8}$ combined with any one of three $\mathbf{3 5}$ generate an $\mathfrak{s u}(8)$ subalgebra of $\mathfrak{e}_{7}$. Compact $\mathfrak{s o}(8)$ becomes $\mathfrak{s o}^{*}(8)$ if we consider $\mathfrak{e}_{7(-25)}$ instead of compact $\mathfrak{e}_{7}$ and $\mathfrak{s u}(8)$ becomes $\mathfrak{s u}^{*}(8)$.

Consider the Clifford algebra of $\mathbb{R}^{6,2}$

$$
\left\{\Gamma^{a}, \Gamma^{b}\right\}=2 \eta^{a b}
$$

and choose a basis with the following hermiticity property

$$
\left(\Gamma^{a}\right)^{\dagger}=\eta_{a b} \Gamma^{b}=\omega \cdot \Gamma^{a} \cdot \omega^{-1}
$$

where $\omega=\Gamma^{7} \cdot \Gamma^{8}$ is a $16 \times 16$ symplectic matrix. One particular choice of basis, in which chirality matrix $\Gamma^{9}$ is diagonal, is given as follows

$$
\begin{array}{lr}
\Gamma^{1}=\sigma_{1} \otimes \mathbb{I}_{2} \otimes \mathbb{I}_{2} \otimes \mathbb{I}_{2} & \Gamma^{2}=\sigma_{2} \otimes \sigma_{1} \otimes \mathbb{I}_{2} \otimes \sigma_{2} \\
\Gamma^{3}=\sigma_{2} \otimes \sigma_{2} \otimes \sigma_{2} \otimes \sigma_{2} & \Gamma^{4}=\sigma_{2} \otimes \sigma_{2} \otimes \sigma_{3} \otimes \mathbb{I}_{2} \\
\Gamma^{5}=\sigma_{2} \otimes \sigma_{3} \otimes \mathbb{I}_{2} \otimes \sigma_{2} & \Gamma^{6}=\sigma_{2} \otimes \sigma_{2} \otimes \sigma_{1} \otimes \mathbb{I}_{2} \\
\Gamma^{7}=i \sigma_{2} \otimes \mathbb{I}_{2} \otimes \sigma_{2} \otimes \sigma_{3} & \Gamma^{8}=i \sigma_{2} \otimes \mathbb{I}_{2} \otimes \sigma_{2} \otimes \sigma_{1}
\end{array}
$$

\footnotetext{
${ }^{6}$ Notice that compact $\mathfrak{s u}(8)$ is not a subalgebra of $\mathfrak{e}_{7(-25)}$.
} 
Then,

$$
\begin{aligned}
Z^{a b} & =\frac{1}{4} \Gamma^{a b}{ }_{C D}\left(X^{C D}-i P_{C D}\right) \\
\tilde{Z}^{a b} & =\frac{1}{4} \Gamma^{a b}{ }_{C D}\left(X^{C D}+i P_{C D}\right)
\end{aligned}
$$

where transformation coefficient are given by matrix elements of chiral representation of $\mathfrak{s o}(6,2)$ generators

$$
\Gamma_{C D}^{a b}=\mathbb{P}\left(\frac{i}{4}\left[\Gamma^{a}, \Gamma^{b}\right]\right)_{C D}
$$

and $\mathbb{P}$ is the chiral projection operator in spinor space. Symplectic structure (20) of $X$ and $P$ induces the symplectic structure

$$
\left[\tilde{Z}^{a b}, Z^{c d}\right]=\frac{1}{8} \operatorname{Tr}\left[\Gamma^{a b} \Gamma^{c d}\right]=\frac{1}{2}\left(\eta^{c a} \eta^{d b}-\eta^{c b} \eta^{d a}\right) .
$$

on $Z$ and $\tilde{Z}$. Gamma matrices defined above satisfy the following identities

$$
\begin{aligned}
& \Gamma_{A B}^{a b}=-\Gamma_{B A}^{a b}=-\Gamma^{b a}{ }_{A B} \\
& \Gamma_{A B}^{a b c d}=\frac{1}{24} \epsilon^{a b c d}{ }_{\text {efgh }} \Gamma_{A B}^{e f g h}=\Gamma_{B A}^{a b c d} \\
& \left.\Gamma^{a b c d}{ }_{A B C D}:=\Gamma^{[a b}{ }_{[A B} \Gamma^{c d]}{ }_{C D}\right] \\
& \Gamma_{A B C D}^{a b c d}=-\frac{1}{24} \epsilon_{\text {efgh }}^{a b c d} \Gamma_{A B C D}^{e f g h}=-\frac{1}{24} \epsilon_{A B C D E F G H} \Gamma_{E F G H}^{a b c d} \\
& \left.\Gamma_{[A B}^{a c} \Gamma_{C D]}^{c b}=\Gamma_{[A B}^{b c} \Gamma^{c a}{ }_{C D]}=\frac{1}{24} \epsilon_{A B C D E F G H} \Gamma^{a c}{ }_{[E F} \Gamma^{c b}{ }_{G H}\right]
\end{aligned}
$$

where

$$
\Gamma_{A B}^{a b c d}=\mathbb{P}\left(\Gamma^{[a} \Gamma^{b} \Gamma^{c} \Gamma^{d]}\right)_{A B} .
$$

These identities allow us to rewrite generators of $\mathfrak{e}_{7(-25)}$ in $\mathfrak{s u}(6,2)$ basis:

$$
\begin{aligned}
\eta^{b c} J_{c}^{a}-\eta^{a c} J_{c}^{b} & =\Gamma_{A B}^{a b}\left(J_{B}^{A}-J_{A}^{B}\right) \\
\eta^{b c} J_{c}^{a}+\eta^{a c} J_{c}^{b} & =\Gamma_{A B C D}^{a b}\left(J^{A B C D}+(\epsilon J)_{A B C D}\right) \\
J^{a b c d}+\frac{1}{24} \epsilon^{a b c d}{ }_{e f g h} J^{e f g h} & =\Gamma_{A B}^{a b c d}\left(J_{B}^{A}+J_{A}^{B}\right) \\
J^{a b c d}-\frac{1}{24} \epsilon^{a b c d}{ }_{e f g h} J^{e f g h} & =\Gamma_{A B C D}^{a b c d}\left(J^{A B C D}-(\epsilon J)_{A B C D}\right)
\end{aligned}
$$

or, more succintly,

$$
\begin{aligned}
\eta^{b c} J_{c}^{a} & =\Gamma_{A B}^{a b} J_{B}^{A}+\Gamma_{A B C D}^{a b} J^{A B C D} \\
J^{a b c d} & =\Gamma_{A B}^{a b c d} J_{B}+\Gamma_{A B C D}^{a b c d} J^{A B C D}
\end{aligned}
$$




\section{B Minimal realization of $\mathfrak{e}_{8(8)}$ in $\mathfrak{s u}^{*}(8)$ basis}

Non-compact exceptional Lie algebra $\mathfrak{e}_{8(8)}$ also admits realization in an $\mathfrak{s u}^{*}(8)$ basis. It is seen via the following chain of subalgebra inclusions $\mathfrak{s u}^{*}(8) \subset \mathfrak{e}_{7(7)} \subset$ $\mathfrak{e}_{8(8)}$.

Algebra $\mathfrak{e}_{7(7)}$ is generated as

$$
\begin{aligned}
J^{A}{ }_{B} & =-2 i X^{A C} P_{C B}-\frac{i}{4} \delta^{A}{ }_{B} X^{C D} P_{C D} \\
J^{A B C D} & =-\frac{i}{2} X^{[A B} X^{C D]}+\frac{i}{48} \epsilon^{A B C D E F G H} P_{E F} P_{G H} .
\end{aligned}
$$

where $A, B, \ldots$ are $\mathfrak{s u}^{*}(8)$ indices. Note different relative signs between $X X$ and $P P$ terms in (112) and (24). It amounts to change of sign in the commutator on the third line

$$
\begin{aligned}
{\left[J_{B}^{A}, J^{C}{ }_{D}\right] } & =\delta^{C}{ }_{B} J^{A}{ }_{D}-\delta^{A}{ }_{D} J^{C}{ }_{B} \\
{\left[J^{A}{ }_{B}, J^{C D E F}\right] } & =-4 \delta^{[C}{ }_{B} J^{D E F] A}-\frac{1}{2} \delta^{A}{ }_{B} J^{C D E F} \\
{\left[J^{A B C D}, J^{E F G H}\right] } & =+\frac{1}{36} \epsilon^{A B C D K[E F G} J^{H]} K
\end{aligned}
$$

as compared to that in (3) while does not change the hermiticity properties (4) resulting in the following quadratic Casimir

$$
\begin{aligned}
\mathcal{C}_{2} & =\frac{1}{6} J_{B}^{A} J_{A}^{B}+\frac{1}{24} \epsilon_{A B C D E F G H} J^{A B C D} J^{E F G H} \\
& =\frac{1}{6} J_{B}^{A} J_{A}^{B}+J^{A B C D}(\epsilon J)_{A B C D} .
\end{aligned}
$$

The decomposition of $\mathfrak{e}_{7(7)}$ with respect to the maximal compact subalgebra $\mathfrak{u s p}(8)$ of $\mathfrak{s u}^{*}(8)$ results now in

$$
133=63 \oplus \mathbf{7 0}=\left(\mathbf{3 6}_{\text {c. }} \oplus \mathbf{2} \mathbf{7}_{\text {n.c. }}\right) \oplus\left(\mathbf{4} \mathbf{2}_{\text {n.c. }} \oplus \mathbf{2} \mathbf{7}_{\text {c. }} \oplus \mathbf{1}_{\text {n.c. }}\right)
$$

and shows the the constructed $\mathfrak{e}_{7}$ is indeed $\mathfrak{e}_{7(7)}$. The remaining generators of algebra $\mathfrak{e}_{8(8)}$ are then given by

$$
E^{A B}=-i y X^{A B} \quad \tilde{E}_{A B}=-i y P_{A B} \quad E=-\frac{i}{2} y^{2}
$$

and

$$
\begin{array}{ll}
F=\frac{1}{2 i} p^{2}+\frac{2}{i y^{2}} I_{4}(X, P) & F^{A B}=i p X^{A B}+\frac{2}{y}\left[X^{A B}, I_{4}(X, P)\right] \\
I_{4}(X, P)=\mathcal{C}_{2}+\frac{323}{16} & \tilde{F}_{A B}=i p P_{A B}+\frac{2}{y}\left[P_{A B}, I_{4}(X, P)\right] .
\end{array}
$$


They satisfy the same commutation relations as their counterparts of $\mathfrak{e}_{8(-24)}$ except for

$$
\begin{aligned}
{\left[J^{A B C D}, E^{E F}\right] } & =+\frac{1}{24} \epsilon^{A B C D E F G H} \tilde{E}_{G H} \\
{\left[J^{A B C D}, F^{E F}\right] } & =+\frac{1}{24} \epsilon^{A B C D E F G H} \tilde{F}_{G H} \\
{\left[\tilde{E}_{A B}, \tilde{F}_{C D}\right] } & =+12(\epsilon J)_{A B C D}
\end{aligned}
$$

\section{References}

[1] A. Joseph, "Minimal realizations and spectrum generating algebras", Commun. Math. Phys. 36, 325 (1974)

[2] Y. Dothan, M. Gell-Mann and Y. Ne'eman, Physics Letters 17 ( 1965) 148; A.O.Barut, "Applications of the dynamical group theory to the structure of hadrons", in Lectures in Theoretical Physics eds. A.O.Barut and W.E.Brittin, Vol. 10B , pp 377-426.

[3] A. Joseph. "The minimal orbit in a simple Lie algebra and its associated maximal ideal", Ann. Sci. Ec. Norm. Super., IV. Ser. 9, 1 (1976)

[4] Jian-Shu Li , " Minimal representations and reductive dual pairs", in "Representation Theory of Lie Groups" , IAS/Park City Mathematics Series Volume 8, eds. J. Adams and D. Vogan, AMS Publications (2000).

[5] D. Kazhdan, B. Pioline and A. Waldron, "Minimal representations, spherical vectors, and exceptional theta series. I," Commun. Math. Phys. 226, 1 (2002) arXiv:hep-th/0107222.

[6] M. Gunaydin, K. Koepsell and H. Nicolai, "The Minimal Unitary Representation of $\mathrm{E}_{8(8)}, "$ Adv. Theor. Math. Phys. 5, 923 (2002) arXiv:hep-th/0109005.

[7] B. Pioline and A. Waldron, " The automorphic membrane", [ arXiv:hep-th/0404018.

[8] M. Gunaydin, K. Koepsell and H. Nicolai, "Conformal and quasiconformal realizations of exceptional Lie groups," Commun. Math. Phys. 221, 57 (2001) arXiv:hep-th/0008063.

[9] E. Cremmer , B. Julia and J. Scherk, " Supergravity Theory in Eleven Dimensions", Phys. Lett. 76B, (1978) 409.

[10] M. Gunaydin, G. Sierra and P. K. Townsend, "The Geometry Of N=2 Maxwell-Einstein Supergravity And Jordan Algebras," Nucl. Phys. B 242, 244 (1984). 
[11] R. Brylinski and B. Konstant, "Minimal representations of $E_{6}, E_{7}$ and $E_{8}$ and the generalized Capelli identity," Proc. Nat. Acad. Sci. U.S.A. 91, 2469 (1994); "Minimal representations, geometric quantization and unitarity," Proc. Nat. Acad. Sci. U.S.A. 916026 (1994); "Lagrangian models of minimal representations of $E_{6}, E_{7}$ and $E_{8}$," in "Functional Analysis on the Eve of 21st century", Progress in Math., Birkhäuser (1995).

[12] R. Gilmore, "Lie Groups Lie Algebras and Some of Their Applications", Kieger Pub. Co ( 1974)

[13] W.G. McKay and J. Patera," Tables of dimensions, indices, and branching rules for representations of simple Lie algebras", Marcel Dekker, Inc. ( New York ,1981)

[14] M. Gunaydin and O. Pavlyk, in preparation.

[15] V. de Alfaro, S. Fubini and G. Furlan, " Conformal invariance in quantum mechanics", Nuovo Cimento 34A, (1976) 569.

[16] M. Gunaydin, " Generalized conformal and superconformal group actions and Jordan algebras", Mod. Phys. Lett . A8 (1993) 1407. 